Pacific Journal of Mathematics

LATTICES OF COMPLETELY REGULAR SEMIGROUP 


\title{
LATTICES OF COMPLETELY REGULAR SEMIGROUP VARIETIES
}

\author{
F. J. Pastijn and P. G. Trotter
}

\begin{abstract}
Let $\rho$ be a fully invariant congruence on the free completely regular semigroup $F_{X}^{\mathrm{CR}}$ of countably infinite rank. Let $\rho_{\min }$ and $\rho^{\mathrm{min}}$ be the least congruences on $F_{X}^{\mathrm{CR}}$ with respectively the same trace and the same kernel as $\rho$. Let $\rho_{\max }$ and $\rho^{\max }$ be the greatest congruences on $F_{X}^{\mathrm{CR}}$ with respectively the same trace and the same kernel as $\rho$. These congruences are shown to be fully invariant. We construct a network of varieties corresponding to the congruences $\cdots, \rho^{\max }, \rho_{\max }, \rho, \rho^{\min }, \rho_{\min },\left(\rho_{\min }\right)^{\min }$, $\left(\rho^{\min }\right)_{\min }, \cdots$ and their intersections. Intervals between successive joins of the network, in the lattice of subvarieties of completely regular semigroups, are characterised as direct products of particular subintervals. By comparing the network with the chain of varieties that are each generated by a free completely regular semigroup of finite rank we get information on the network and the chain.
\end{abstract}

1. Introduction. Completely regular semigroups are semigroups that are unions of their subgroups. They may be regarded as universal algebras with an associative binary operation (multiplication) and a unary operation (inversion). As universal algebras, completely regular semigroups form a variety determined by the identities

$$
x=x x^{-1} x, \quad x x^{-1}=x^{-1} x, \quad\left(x^{-1}\right)^{-1}=x .
$$

Let $\mathbf{C R}$ denote this variety and $L_{\mathbf{C R}}$ denote the lattice of subvarieties of CR.

Descriptions of sublattices of $L_{\mathbf{C R}}$ have proliferated in recent years. The sublattices for which detailed results have been obtained are all bounded above by BG $\vee \mathbf{O}$ where BG denotes the variety of all bands of groups and $\mathbf{O}$ denotes the variety of all orthodox completely regular semigroups. We will see that these sublattices lie at the bottom of $L_{\mathbf{C R}}$. Varieties under BG $\vee \mathbf{O}$ include the variety $\mathbf{G}$ of all groups, the variety $\mathbf{B}$ of all bands and the variety $\mathbf{C S}$ of all completely simple semigroups. The lattice of subvarieties of $\mathbf{B}$ has been fully described in [1], [6] and [7]. The lattice of subvarieties of CS has been studied in [17], [18], [21], [28] and [31] while other sublattices of $L_{\mathbf{C R}}$ have been investigated in [15], [19], [24], [25], [27], [30] and [22]. 
We will see in the final section of the paper that if $\rho$ is the universal congruence on $F_{X}^{\text {CR }}$ then the varieties induced by $\rho_{\min }, \rho^{\min },\left(\rho^{\min }\right)_{\min }$ and $\left(\rho_{\text {min }}\right)^{\text {min }}$ respectively are $\mathbf{G}, \mathbf{B}, \mathbf{B G}$ and $\mathbf{O}$. The variety $\mathbf{B G} \vee \mathbf{O}$ is induced by $\left(\rho^{\min }\right)_{\min } \cap\left(\rho_{\min }\right)^{\min }$ and is therefore near the bottom of our network.

The paper begins with preliminary notions and with two results on fully invariant congruences. Basic properties of $\rho_{\min }, \rho^{\min }, \rho_{\max }$ and $\rho^{\max }$, where $\rho$ is a congruence on a regular semigroup $S$, are derived in $\S 3$. The min-network $\rho, \rho_{\min }, \rho^{\min },\left(\rho^{\min }\right)_{\min },\left(\rho_{\min }\right)^{\min }, \ldots$, with intersections, is shown to be a sublattice of the lattice $\mathscr{C}(S)$ of all congruences on $S$; this generalises a construction of M. Petrich and N. R. Reilly [29] based on the universal congruence of an inverse semigroup.

In $\S 4$ we see that if a congruence $\rho$ on $F_{X}^{\text {CR }}$ is fully invariant then so is $\rho_{\min }, \rho^{\min }, \rho_{\max }$ and $\rho^{\max }$. We see in $\$ 5$ that the max-network for a fully invariant congruence is a sublattice of $\mathscr{C}\left(F_{X}^{\mathrm{CR}}\right)$. The major result concerning the congruence network for a fully invariant congruence $\rho$ on $F_{X}^{\mathbf{C R}}$ is that, as intervals of the lattice of fully invariant congruences on $F_{X}^{\mathbf{C R}}$,

$$
\begin{aligned}
{\left[\rho_{\min } \cap \rho^{\min }, \rho\right] } & \cong\left[\rho_{\min }, \rho\right] \times\left[\rho^{\min }, \rho\right] \\
& \cong\left[\rho_{\min } \cap \rho^{\min }, \rho_{\min }\right] \times\left[\rho_{\min } \cap \rho^{\min }, \rho^{\min }\right]
\end{aligned}
$$

The corresponding result holds for $\left[\rho, \rho_{\max } \vee \rho^{\max }\right]$. We restate these results in varietal terminology and compare them with some results from the literature. If $\rho$ is not the equality relation then our network of varieties is infinite with supremum $\mathbf{C R}$.

We also prove that for distinct integers $m$ and $n$ the free completely regular semigroups of ranks $m$ and $n$ generate distinct varieties. The free completely regular semigroups of finite rank are not fundamental while $F_{X}^{\mathbf{C R}}$ is fundamental.

For undefined notation or terminology see [11] or [16].

2. Definitions and preliminary results. Let $S$ be a regular semigroup. Denote by $E(S)$ the set of idempotents of $S$ and by $V(x)$ the set of inverses of $x \in S$. We make use in the text of Nambooripad's sandwich set $S(e, f)$ for $e, f \in E(S)$ (see [22] or [23]). Specifically

$$
S(e, f)=\{p \in E(S) ; p e=p=f p, e p f=e f\} .
$$

We note that $S(e, f) \subseteq E(S) \cap V(e f)$.

The description in [10] and [38] of the free completely regular semigroup $F_{X}^{\mathbf{C R}}$ on a set $X$ is via a description of the free unary semigroup $F_{X}^{\mathrm{U}}$ on $X$; that is, the free object on $X$ in the variety of all semigroups with a unary operation. Let $\bar{X}=X \cup\left\{(,)^{-1}\right\}$ where $(,)^{-1} \notin X$. By [9], $F_{X}^{\mathbf{U}}$ is 
the smallest subsemigroup of the free semigroup $F_{\bar{X}}$ on $\bar{X}$ such that $X \subseteq F_{X}^{\mathbf{U}}$ and $(w)^{-1} \in F_{X}^{\mathbf{U}}$ for all $w \in F_{X}^{\mathbf{U}}$. Define the content of $w \in F_{\bar{X}}$ to be the set

$$
c(w)=\{\text { letters of } X \text { appearing in } w\} .
$$

If $u=b(a \text { or } u=a)^{-1} b$ where $a \in\left(F_{X}^{\mathrm{U}}\right)^{1}$ and $b \in F_{\bar{X}}^{1}$ we say that the occurrence of ( or $)^{-1}$ respectively is unmatched. If $v \in F_{\bar{X}}$ let $\bar{v} \in F_{X}^{\mathrm{U}}$ denote the word obtained from $v$ by successively deleting unmatched occurrences of ( and $)^{-1}$.

Define the left indicator $L(w)$ of $w \in F_{X}^{\mathbf{U}}$ by $L(w)=\bar{a}$ where $a$ is the shortest initial segment of $w$ such that $c(a)=c(w)$. Similarly the right indicator of $w$ is $R(w)=\tilde{b}$ where $b$ is the shortest final segment of $w$ such that $c(w)=c(b)$. It follows that $L(w)=u x$ for some $u \in\left(F_{X}^{\mathrm{U}}\right)^{1}$ and $x \in X \backslash c(u)$ and $R(w)=y v$ for some $v \in\left(F_{X}^{\mathbf{U}}\right)^{1}$ and $y \in X \backslash c(v)$.

As a notational convenience write $w^{-1}$ in place of $(w)^{-1}$ in $F_{X}^{\mathbf{U}}$. Let $\lambda$ be the congruence on $F_{X}^{U}$ generated by the pairs $\left(w, w w^{-1} w\right),\left(w w^{-1}, w^{-1} w\right)$ and $\left(\left(w^{-1}\right)^{-1}, w\right)$ for all $w \in F_{X}^{U}$. The following summarizes Theorems 3.1, 4.2 and 5.4 of $[2]$

THEOREM 2.1 [2]. Let $u, v \in F_{X}^{\mathrm{U}}$. Then

(i) $F_{X}^{\mathbf{C R}} \cong F_{X}^{\mathbf{U}} / \lambda$.

(ii) $u \lambda \mathscr{D} v \lambda$ if and only if $c(u)=c(v)$, and

(iii) $u \lambda \mathscr{R} v \lambda$ if and only if $L(u)=a x$ and $L(v)=b x$ for some $x \in X$ and $a, b \in\left(F_{X}^{\mathbf{U}}\right)^{1}$ such that $(a, b) \in \lambda$ or $a=b=1$.

In the remainder of the paper we assume $X$ is a countably infinite set. Every element of $F_{X}^{\mathrm{CR}}$ can be written in the form $w$ with $w \in F_{X}^{\mathrm{U}}$. We hereby assume that $w=v$ in $F_{X}^{\mathrm{CR}}$ if and only if the identity $w=v$ is a consequence of the identities $x x^{-1} x=x, x x^{-1}=x^{-1} x$ and $\left(x^{-1}\right)^{-1}=x$. Accordinly if $\mathbf{V}$ is a subvariety of the variety $\mathbf{C R}$ of all completely regular semigroups then we shall write the identities that hold in $\mathbf{V}$ in the form $w=v$ where $w, v \in F_{X}^{\mathbf{U}}$.

A binary relation $\theta$ on a semigroup $S$ is said to be fully invariant if for every endomorphism $\phi$ of $S$ and every pair $(x, y) \in \theta$ then $(x \phi, y \phi) \in \theta$.

LEMMA 2.2. Let $S$ be a semigroup and $\theta$ be a fully invariant reflexive, symmetric binary relation on $S$. Then the congruence $\rho$ on $S$ that is generated by $\theta$ is fully invariant.

Proof. By [35] $(a, b) \in \rho$ if and only if there exist $p_{i}, q_{i} \in S^{1}$ and $\left(a_{i}, b_{i}\right) \in \theta$ for $1 \leq i \leq n$, some $n$, such that

$$
a=p_{1} a_{1} q_{1}, \quad p_{1} b_{1} q_{1}=p_{2} a_{2} q_{2}, \ldots, \quad p_{n} b_{n} q_{n}=b .
$$


Let $\phi$ be an endomorphism of $S$ and put $1 \phi=1$. Suppose $(a, b) \in \rho$. Then $\left(p_{i} b_{i} q_{i}\right) \phi=\left(p_{i+1} a_{i+1} q_{i+1}\right) \phi$ and, since $\theta$ is fully invariant, $\left(a_{i} \phi, b_{i} \phi\right) \in \theta$ for all $i$. So $(a \phi, b \phi) \in \rho$.

LEMMA 2.3. Let $\mathscr{S}$ be a category of semigroups with a free object $F_{X}^{\mathscr{S}}$ on a countably infinite set $X$. Let $\theta$ be a fully invariant equivalence relation on $F_{X}^{\mathscr{S}}$. Then the largest congruence $\rho$ contained in $\theta$ is fully invariant.

Proof. By [3; Lemma 10.3] we have

$$
\rho=\left\{(a, b) \in \theta ;(p a q, p b q) \in \boldsymbol{\theta} \text { for all } p, q \in\left(F_{X}^{\mathscr{S}}\right)^{1}\right\} .
$$

Suppose $(a, b) \in \rho$. There exists $x_{1}, \ldots, x_{n} \in X$ such that $a$ and $b$ are products of elements from $\left\{x_{1}, \ldots, x_{n}\right\}$. Select $z_{1}, z_{2} \in(X \cup\{1\}) \backslash$ $\left\{x_{1}, \ldots, x_{n}\right\}$. Let $\phi$ be an endomorphism of $F_{X}^{\mathscr{S}}$ and $p, q \in\left(F_{X}^{\mathscr{S}}\right)^{1}$. Define an endomorphism $\psi$ of $F_{X}^{\mathscr{S}}$ such that

$$
x_{h} \psi=x_{h} \phi \quad \text { for all } h, 1 \leq h \leq n, \quad z_{1} \psi=p \quad \text { and } \quad z_{2} \psi=q .
$$

We assume $p=1$ if and only if $z_{1}=1$ and $q=1$ if and only if $z_{2}=1$. Since $\left(z_{1} a z_{2}, z_{1} b z_{2}\right) \in \theta$ and $\theta$ is fully invariant then $\left(\left(z_{1} a z_{2}\right) \psi,\left(z_{1} b z_{2}\right) \psi\right)$ $\in \theta$, so $(p(a \phi) q, p(b \phi) q) \in \theta$. This follows for all $p, q \in\left(F_{X}^{\mathscr{S}}\right)^{1}$ so $(a \phi, b \phi) \in \rho$.

With the obvious modification to the description of $\rho$ in the proof it can be seen that Lemma 2.3 holds for any variety of algebras of finite type $\tau$.

We note that since completely regular semigroups are unions of groups then surjective semigroup homomorphisms preserve the unary operation. Conversely, unary semigroup homomorphisms are semigroup homomorphisms. Hence we need not distinguish between semigroup congruences and unary semigroup congruences on a completely regular semigroup.

In the text we consider the following lattices and their sublattices; the lattice $\mathscr{C}(S)$ of all congruences on a semigroup $S$, the lattice $C\left(F_{X}^{\mathrm{CR}}\right)$ of all fully invariant congruences on $F_{X}^{\mathbf{C R}}$, and the lattice $L_{\mathbf{C R}}$ of all subvarieties of $\mathbf{C R}$.

If $\rho$ is a fully invariant congruence on $F_{X}^{\mathbf{C R}}$ then denote by $\mathbf{V}_{\rho}$ the subvariety of $\mathbf{C R}$ determined by the identities $w=v$ for all $(w, v) \in \rho$. If $\mathbf{V}$ is a subvariety of $\mathbf{C R}$ denote by $F_{X}^{\mathbf{V}}$ the free object in $\mathbf{V}$ on $X$, and by $\rho_{\mathbf{V}}$ the congruence which is induced on $F_{X}^{\text {CR }}$ by the natural homomorphism $F_{X}^{\mathbf{C R}} \rightarrow F_{X}^{\mathbf{V}}$. Then $\rho_{\mathrm{V}}$ is a fully invariant congruence. Moreover,

$$
L_{\mathbf{C R}} \rightarrow C\left(F_{X}^{\mathbf{C R}}\right) ; \quad \mathbf{V} \rightarrow \rho_{\mathbf{V}} \text { and } C\left(F_{X}^{\mathbf{C R}}\right) \rightarrow L_{\mathbf{C R}} ; \quad \rho \rightarrow \mathbf{V}_{\rho}
$$


are mutually inverse anti-isomorphisms between the lattices $L_{\mathbf{C R}}$ and $C\left(F_{X}^{\mathbf{C R}}\right)$. For more detail see [11, $\left.\S \S 24,25,26\right]$.

3. Congruences on regular semigroups. We make extensive use of the kernel-trace description of congruences on regular semigroups. The description was introduced for inverse semigroups by H. E. Scheiblich [34] and extended to regular semigroups by R. Feigenbaum in [4] and [5]. We use the notation of [26].

Let $S$ be a regular semigroup. Recall that $\mathscr{C}(S)$ denotes the lattice of congruences on $S$. The kernel of a congruence $\rho$ on $S$ is the union of all $\rho$-classes that contain idempotents, that is,

$$
\operatorname{ker} \rho=\{x \in S ; x \rho e \text { for some } e \in E(S)\} \text {. }
$$

The trace of $\rho$ is the restriction of $\rho$ to $E(S)$, thus $\operatorname{tr} \rho=\rho \mid E(S)$. For any congruence $\rho$ define

$$
\begin{aligned}
& K_{\rho}=\{\theta \in \mathscr{C}(S) ; \operatorname{ker} \rho=\operatorname{ker} \theta\}, \\
& T_{\rho}=\{\theta \in \mathscr{C}(S) ; \operatorname{tr} \rho=\operatorname{tr} \theta\} .
\end{aligned}
$$

By [4] and [5; Theorem 4.1] a congruence $\rho$ on $S$ is completely determined by its trace and kernel; in other words

$$
\{\tau\}=K_{\rho} \cap T_{\rho} .
$$

It readily follows that $K_{\rho}$ and $T_{\rho}$ are convex subsets of $\mathscr{C}(S)$.

For $\rho \in \mathscr{C}(S)$ define $\rho_{\min }=\cap T_{\rho}$. Then $\operatorname{tr} \rho_{\min }=\operatorname{tr} \rho$ and $\rho_{\min }$ is the least element of $T_{\rho}$. Hence $\rho_{\min }$ is the congruence on $S$ which is generated by the relation $\operatorname{tr} \rho$. Therefore, if $\rho, \theta \in \mathscr{C}(S)$, then

$$
\operatorname{tr} \rho \subseteq \operatorname{tr} \theta \text { implies } \rho_{\min } \subseteq \theta_{\text {min }} .
$$

Recall the definition of sandwich sets. For $\rho \in \mathscr{C}(S)$ let $\rho^{\min }$ be the congruence on $S$ which is generated by the relation

$$
\left\{(x, e) ; x \in \operatorname{ker} \rho, e \in S\left(x^{\prime} x, x x^{\prime}\right) \text { for some } x^{\prime} \in V(x)\right\} .
$$

It follows from this definition that, for $\theta, \rho \in \mathscr{C}(S)$,

$$
\operatorname{ker} \rho \subseteq \operatorname{ker} \theta \text { implies } \rho^{\min } \subseteq \theta^{\min } \text {. }
$$

If $\theta \in K_{\rho}$ and $x \in \operatorname{ker} \theta=\operatorname{ker} \rho$ then by [36; Lemma 1.2] we have $(x, e) \in \theta$ for every $x^{\prime} \in V(x)$ and $e \in S\left(x^{\prime} x, x x^{\prime}\right)$. Thus $\rho^{\min } \subseteq \theta$ and in particular $\rho^{\min } \subseteq \rho$. Hence, by (2), (5) and (6), ker $\rho^{\min }=\operatorname{ker} \rho$. We have shown that $\rho^{\text {min }}$ is the least element of $K_{\rho}$.

A congruence on $S$ is idempotent separating if its trace is the equality relation on $E(S)$. We see, using [14; Theorem 5], that the greatest 
idempotent separating congruence $\mu_{S}$ on $S$ is characterized by

$$
\begin{aligned}
& \mu_{S}=\left\{(x, y) \in \mathscr{H} ; \text { for some }(\text { any }) x^{\prime} \in V(x)\right. \text { and } \\
& y^{\prime} \in V(y) \text { where }\left(x^{\prime}, y^{\prime}\right) \in \mathscr{H} \text { then } x^{\prime} e x=y^{\prime} e y \\
&\text { for each idempotent } \left.e \leq x x^{\prime}\right\} .
\end{aligned}
$$

Let $\rho \in \mathscr{C}(S)$. Using Lallement's Lemma ([20] and [16; II Lemma 4.6]) we see that a congruence $\theta$ belongs to $T_{\rho}$ if and only if $\rho_{\min } \subseteq \theta$ and $\theta / \rho_{\min }$ is idempotent separating on $S / \rho_{\min }$. Hence the congruence $\rho_{\max }$ on $S$ given by

$$
x \rho_{\max } y \text { if and only if } x \rho^{\sharp} \mu_{S / \rho} y \rho^{\#}
$$

is the greatest element of $T_{\rho}$ (we let $\rho^{\#}: S \rightarrow S / \rho$ denote the natural homomorphism).

LEMMA 3.1. Let $S$ be a regular semigroup and $\rho, \theta \in \mathscr{C}(S)$. Then $\operatorname{tr} \rho \subseteq \operatorname{tr} \theta$ implies $\rho_{\max } \subseteq \boldsymbol{\theta}_{\max }$.

Proof. For any congruence $\sigma$ on $S$ and $a, b \in S$ define an equivalence relation $\mathscr{H}_{\text {tr } \sigma}$ by

$$
a \mathscr{H}_{\mathrm{tr} \sigma} b \text { if and only if } a\left(\sigma_{\text {min }}\right)^{\#} \mathscr{H} b\left(\sigma_{\text {min }}\right)^{\#} \text { in } S / \sigma_{\text {min }} .
$$

Let $\mu$ be the greatest congruence on $S$ that is contained in $\mathscr{H}_{\operatorname{tr} \sigma}$. Since $\mu \supseteq \sigma_{\min }$ then $\mu / \sigma_{\min }$ is the greatest congruence on $S / \sigma_{\min }$ that is contained in $\mathscr{H}$; hence $\mu / \sigma_{\min }=\mu_{S / \sigma_{\operatorname{mim}}}$. So by (8) and since $\operatorname{tr} \sigma_{\min }=\operatorname{tr} \sigma$ then $\mu=\left(\sigma_{\min }\right)_{\max }=\sigma_{\max }$. We have $\operatorname{tr} \rho \subseteq \operatorname{tr} \theta$ so $\rho_{\min } \subseteq \theta_{\min }$ and hence $\mathscr{H}_{\operatorname{tr} \rho} \subseteq$ $\mathscr{H}_{\text {tr } \theta}$. It follows that $\rho_{\max } \subseteq \theta_{\max }$.

For $\rho \in \mathscr{C}(S)$ let $\rho^{\max }$ be the congruence on $S$ given by

$$
\begin{array}{r}
\rho^{\max }=\left\{(x, y) \in S \times S ; \text { for every } p, q \in S^{1},\right. \\
p x q \in \operatorname{ker} \rho \Leftrightarrow p y q \in \operatorname{ker} \rho\} .
\end{array}
$$

By [35] $\rho^{\max }$ is the greatest congruence such that $\operatorname{ker} \rho$ is a union of its classes. It readily follows that $\rho^{\max }$ is the greatest element of $K_{\rho}$. A congruence on $S$ is idempotent pure if its kernel coincides with $E(S)$. By Lallement's Lemma a congruence $\theta$ belongs to $K_{\rho}$ if and only if $\rho^{\min } \subseteq \theta$ and $\theta / \rho^{\min }$ is idempotent pure on $S / \rho^{\min }$.

If $\rho, \theta \in \mathscr{C}(S)$ and $\operatorname{ker} \rho \subseteq \operatorname{ker} \theta$ (or even $\rho \subseteq \theta$ ) it does not follow that $\rho^{\max } \subseteq \theta^{\text {max }}$. To see this consider the inverse semigroup $S$ that is a 
direct product of a non-trivial group $G$ and the two element semilattice $I=\{0,1\}$. Let $\rho$ be the equality relation on $S$ and $\theta$ be the Rees congruence on $S$ which has $G \times\{0\}$ as its non-trivial class. Then $\rho \subseteq \theta$ but $\rho^{\max } \nsubseteq \theta^{\max }$.

The following result summarises part of our findings.

THEOREM 3.2. Let $\rho$ be a congruence on a regular semigroup $S$. Let $K_{\rho}$ and $T_{\rho}$ be given by (3). Then there exist congruences $\rho_{\min }, \rho^{\min }, \rho_{\max }, \rho^{\max }$ such that as intervals of $\mathscr{C}(S)$

$$
T_{\rho}=\left[\rho_{\min }, \rho_{\max }\right] \text { and } K_{\rho}=\left[\rho^{\min }, \rho^{\max }\right] .
$$

If $\rho, \theta \in \mathscr{C}(S)$ then

$\operatorname{tr} \rho \subseteq \operatorname{tr} \theta$ implies $\rho_{\min } \subseteq \theta_{\min }$ and $\rho_{\max } \subseteq \theta_{\max }$,

$\operatorname{ker} \rho \subseteq \operatorname{ker} \theta$ implies $\rho^{\min } \subseteq \theta^{\min }$.

It should be noted that the characterization of $T_{\rho}$ first appeared in [33; Theorem 3.4]. For inverse semigroups $K_{\rho}$ is characterized in [12; Theorem 3.3].

We next generalise a result of Green on inverse semigroups [12; Theorem 3.8].

THEOREM 3.3. If $\rho$ is a congruence on a regular semigroup $S$ then

$$
\rho=\rho_{\min } \vee \rho^{\min }=\rho_{\max } \cap \rho^{\max } .
$$

Proof. Since $\rho_{\min } \subseteq \rho_{\min } \vee \rho^{\min } \subseteq \rho$ then $\rho_{\min } \vee \rho^{\min } \in T_{\rho}$ and since $\rho^{\min } \subseteq \rho_{\min } \vee \rho^{\min } \subseteq \rho$ then $\rho_{\min } \vee \rho^{\min } \in K_{\rho}$. Hence $\rho_{\min } \vee \rho^{\min } \in T_{\rho} \cap$ $K_{\rho}=\{\rho\}$. A dual proof gives the other equality.

THEOREM 3.4. If $\rho$ is a congruence on a regular semigroup $S$ then

$$
\rho_{\min } \cap \rho^{\min }=\left(\rho^{\min }\right)_{\min } \vee\left(\rho_{\min }\right)^{\min } \in T_{\rho^{\min }} \cap K_{\rho_{\min }} .
$$

Proof. Since $\rho_{\min } \subseteq \rho$ and $\left(\rho^{\min }\right)_{\min } \subseteq \rho^{\min }$ then by Theorem 3.2 $\left(\rho_{\min }\right)^{\min } \vee\left(\rho^{\min }\right)_{\min } \subseteq \rho^{\min }$. Similarly since $\rho^{\min } \subseteq \rho$ then $\left(\rho_{\min }\right)^{\min } \vee$ $\left(\rho^{\min }\right)_{\min } \subseteq \rho_{\min }$. Hence $\left(\rho_{\min }\right)^{\min } \vee\left(\rho^{\min }\right)_{\min } \subseteq \rho_{\min } \cap \rho^{\min }$. Since

$$
\left(\rho_{\min }\right)^{\min } \subseteq\left(\rho_{\min }\right)^{\min } \vee\left(\rho^{\min }\right)_{\min } \subseteq \rho_{\min } \cap \rho^{\min } \subseteq \rho_{\min }
$$

it follows that $\left(\rho_{\min }\right)^{\min } \vee\left(\rho^{\min }\right)_{\min }$ and $\rho_{\min } \cap \rho^{\min }$ are in $K_{\rho_{\min }}$. From

$$
\left(\rho^{\min }\right)_{\min } \subseteq\left(\rho_{\min }\right)^{\min } \vee\left(\rho^{\min }\right)_{\min } \subseteq \rho_{\min } \cap \rho^{\min } \subseteq \rho^{\min }
$$




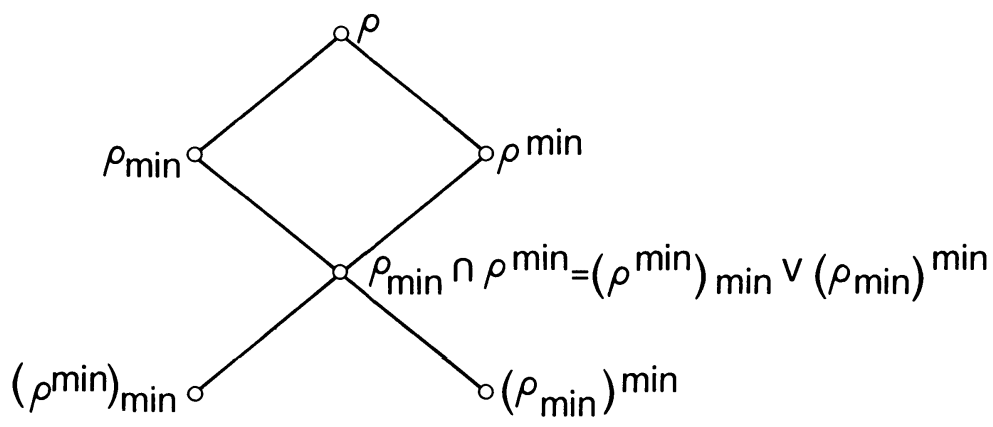

FIGURE 1

it follows that $\left(\rho_{\text {min }}\right)^{\min } \vee\left(\rho^{\min }\right)_{\text {min }}$, and $\rho_{\text {min }} \cap \rho^{\text {min }}$ are in $T_{\rho^{\min }}$. We have proved that $\left(\rho_{\min }\right)^{\min } \vee\left(\rho^{\min }\right)_{\min }$ and $\rho_{\min } \cap \rho^{\min }$ have the same trace and the same kernel; they are equal by (4).

We remark that some of the congruences depicted in Figure 1 may coincide. It will be shown in Theorem 5.12 that when $S=F_{X}^{\mathbf{C R}}$ and $\rho$ is different from the equality relation then $\rho_{\min } \neq\left(\rho^{\min }\right)_{\min }$.

COROLlary 3.5. Let $\rho$ be a congruence on a regular semigroup $S$. Inductively define the congruences $\gamma_{i}, \delta_{l}$ and $\rho_{i}$ for $i \geq 0$ as follows:

$$
\begin{aligned}
\gamma_{0} & =\rho_{\min }, \quad \delta_{0}=\rho^{\min }, \quad \rho_{0}=\rho, \\
\gamma_{i+1} & =\left(\delta_{i}\right)_{\min }, \quad \delta_{i+1}=\left(\gamma_{i}\right)^{\min }, \quad \rho_{\imath}=\gamma_{i} \vee \delta_{i} .
\end{aligned}
$$

Then $\left\{\gamma_{l}, \delta_{i}, \rho_{i} ; i \geq 0\right\}$ forms a sublattice of $\mathscr{C}(S)$ where

$$
\rho_{i} \supseteq \rho_{i+1}, \quad \gamma_{i} \supseteq \gamma_{i+1}, \quad \delta_{i} \supseteq \delta_{i+1}
$$

and

$$
\gamma_{i} \cap \delta_{i}=\gamma_{i+1} \vee \delta_{\imath+1}=\rho_{\imath+1}
$$

If we assume in Corollary 3.5 that the congruences $\rho_{i}$, for all $i \geq 0$, form a infinite chain where $\gamma_{i}$ and $\delta_{i}$ are not comparable then the sublattice of the Corollary can be depicted as in Figure 2. We thus obtain a network of congruences on $S$ that generalises the network of congruences on an inverse semigroup obtained in [29; §5].

We conclude this section with an observation on fully invariant congruences.

THEOREM 3.6. Let $\tau$ be a fully invariant congruence on a regular semigroup $S$. Then $\rho_{\min }$ and $\rho^{\min }$ are fully invariant. 


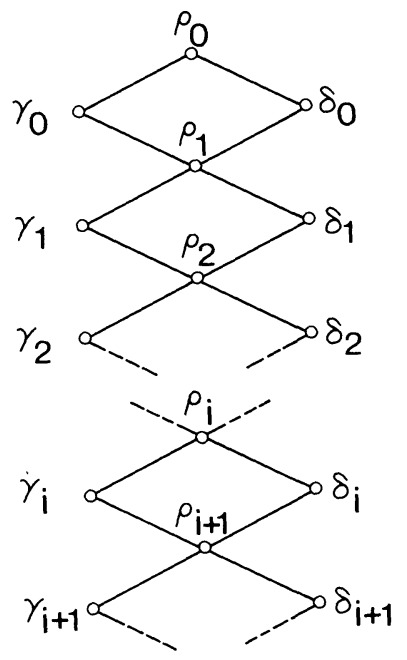

FIGURE 2

Proof. Let $\phi$ be an endomorphism of $S$. Then $\phi$ maps $E(S)$ into $E(S)$. It follows, since $\rho$ is fully invariant, that $\operatorname{tr} \rho$ is a fully invariant relation on $S$. Since $a \in \operatorname{ker} \rho$ if and only if there exists $e \in E(S)$ such that $(a, e) \in \rho$ and since $\rho$ is fully invariant it also follows that $\phi$ maps $\operatorname{ker} \rho$ into $\operatorname{ker} \rho$. Hence, from $\left(S\left(x^{\prime} x, x x^{\prime}\right)\right) \phi \subseteq S\left(\left(x^{\prime} x\right) \phi,\left(x x^{\prime}\right) \phi\right)$ for any $x \in S$, we see that relation (5) is fully invariant. The congruences $\rho_{\min }$ and $\rho^{\min }$ are respectively generated by $\operatorname{tr} \rho$ and relation (5). Hence by Lemma $2.2, \rho_{\min }$ and $\rho^{\min }$ are fully invariant.

4. Fully invariant congruences on a free completely regular semigroup. In the following $X$ will be a countably infinite set and $F_{X}^{\text {CR }}$ the free completely regular semigroup on $X$. Recall that $\mathbf{V}_{\rho}$ is the variety of completely regular semigroups determined by the fully invariant congruence $\rho$ on $F_{X}^{\mathbf{C R}}$. The free object in $\mathbf{V}_{\rho}$ on $X$ is $F_{X}^{\mathbf{V}_{\rho}}=F_{X}^{\mathbf{C R}} / \rho$

THEOREM 4.1. Let $\rho$ be a fully invariant congruence on $F_{X}^{\mathrm{CR}}$. The subvariety $\mathbf{V}_{\rho_{\min }}$ of $\mathbf{C R}$ is determined by all of the identities of the form $w w^{-1}=v v^{-1}$ which hold in $\mathbf{V}_{\rho}$. The subvariety $\mathbf{V}_{\rho_{\min }}$ of $\mathbf{C R}$ is determined by all of the identities of the form $w^{2}=w$ which hold in $\mathbf{V}_{\rho}$.

Proof. By Theorem $3.6 \rho_{\min }$ and $\rho^{\min }$ are fully invariant. The congruence $\rho_{\min }$ on $F_{X}^{\mathbf{C R}}$ is generated by $\operatorname{tr} \rho=\left\{\left(w w^{-1}, v v^{-1}\right) \in \rho ; w, v \in\right.$ $\left.F_{X}^{\mathbf{C R}}\right\}$; hence the description of $\mathbf{V}_{\rho_{\min }}$. Since $\rho^{\min }$ is the least congruence with the same kernel as $\rho$ and since $w \in \operatorname{ker} \rho$ if and only if $\left(w, w^{2}\right) \in \rho$ then the description of $\mathbf{V}_{\rho^{\min }}$ follows. 
THEOREM 4.2. Let $\rho$ be a fully invariant congruence on $F_{X}^{\mathrm{CR}}$. Then $\rho_{\max }$ is a fully invariant congruence on $F_{X}^{\mathbf{C R}}$. The identity $w=v$ holds in $\mathbf{V}_{\rho_{\max }}$ if and only if $(p w q) \rho^{\#} \mathscr{H}(p v q) \rho^{\#}$ in $F_{X^{\rho}}^{\mathbf{X}}$ for all $p, q \in\left(F_{X}^{\mathbf{C R}}\right)^{1}$.

Proof. Let $\phi$ be an endomorphism of $F_{X}^{\mathrm{CR}}$ and $T=F_{X}^{\mathrm{CR}} / \rho$. Since $\rho$ is fully invariant we can define an endomorphism $\psi$ of $T$ by $x \rho^{\#} \psi=x \phi \rho^{\#}$. It is easy to check that the equivalence relation $\mathscr{H}$ on a semigroup is fully invariant. Since the greatest idempotent separating congruence $\mu_{T}$ on $T$ is the greatest congruence under the $\mathscr{H}$-relation on $T$ then by Lemma $2.3 \mu_{T}$ is fully invariant. In particular $\mu_{T}$ is invariant under $\psi$, so by (8) $\rho_{\max }$ is invariant under $\phi$. Thus $\rho_{\max }$ is fully invariant. Since $\mu_{T}$ is greatest under $\mathscr{H}$ then by [3; Lemma 10.3]

$$
\begin{array}{r}
\mu_{T}=\left\{\left(w \rho^{\sharp}, v \rho^{\sharp}\right) ; w, v \in F_{X}^{\mathbf{C R}} \text { such that }(p w q) \rho^{\sharp} \mathscr{H}(p v q) \rho^{\sharp}\right. \\
\text { for all } \left.p, q \in\left(F_{X}^{\mathbf{C R}}\right)^{1}\right\} .
\end{array}
$$

Since $T=F_{X}^{\mathbf{C R}} / \rho \cong F_{X^{\rho}}^{\mathbf{V}^{\rho}}$ then the description of $\rho_{\max }$ follows by (8).

We remark that the proof of Theorem 4.2 shows that for any category of regular semigroups in which free objects exist and on which $\rho$ is a fully invariant congruence, then $\rho_{\max }$ is fully invariant.

As a notational convenience if $S$ is a completely regular semigroup and $w \in S$, then we write $w^{0}=w w^{-1}=w^{-1} w$.

THEOREM 4.3. Let $\rho$ be a fully invariant congruence on $F_{X}^{\mathbf{C R}}$. Then $\rho^{\max }$ is a fully invariant congruence on $F_{X}^{\mathbf{C R}}$. The identity $w=v$ holds in $\mathbf{V}_{\rho^{\max }}$ if and only if, for each $p, q \in\left(F_{X}^{\mathbf{C R}}\right)^{1}$, the following are elements of $\operatorname{ker} \rho$;

(i) $p w q(p v q)^{-1}$,

(ii) $p v q(p w q)^{-1}$,

(iii) $(p w q)^{0}(p v q)^{0}$ and

(iv) $(p v q)^{0}(p w q)^{0}$.

Proof. Let $\tau$ be the relation on $F_{X}^{\mathbf{C R}}$ consisting of the pairs $(w, v)$ such that for each $p, q \in\left(F_{X}^{\mathbf{C R}}\right)^{1}$ the products (i), (ii), (iii) and (iv) are in ker $\rho$. Let $\tau^{T}$ denote the transitive closure of $\tau$. It can be easily seen that $\tau^{T}$ is a congruence on $F_{X}^{\mathbf{C R}}$.

We now check that $\tau^{T}$ is a fully invariant congruence on $F_{X}^{\mathbf{C R}}$. Suppose $(w, v) \in \tau$ and select $x, y \in X \backslash(c(w) \cup c(v))$. Then $x w y(x v y)^{-1}$, $x v y(x w y)^{-1},(x w y)^{0}(x v y)^{0}$ and $(x v y)^{0}(x w y)^{0}$ are in $\operatorname{ker} \rho$. Let $\phi$ be an endomorphism of $F_{X}^{\mathbf{C R}}$. There exists an endomorphism $\psi$ of $F_{X}^{\mathbf{C R}}$ such that 
for any $p, q \in F_{X}^{C R}$ then $z \psi=z \phi$ for all $z \in c(w) \cup c(v), x \psi=p$ and $y \psi=q$. In the proof of Theorem 3.6 we showed that endomorphisms map $\operatorname{ker} \rho$ into $\operatorname{ker} \rho$, so we have $p(w \phi) q(p(v \phi) q)^{-1}, p(v \phi) q(p(w \phi) q)^{-1}$, $(p(w \phi) q)^{0}(p(v \phi) q)^{0}$ and $(p(v \phi) q)^{0}(p(w \phi) q)^{0}$ in ker $\rho$. We may choose $p$ or $q$ to be 1 ; in such cases let $x$ or $y$ respectively be 1 . Hence $(w \phi, v \phi) \in \tau$. So $\tau$ and $\tau^{T}$ are fully invariant.

Say $(w, v) \in \rho$. Then $w v^{-1}, v w^{-1}, w^{0} v^{0}$ and $v^{0} w^{0}$ are in $\operatorname{ker} \rho$; this follows since $u \in \operatorname{ker} \rho$ if and only if $u \rho^{\sharp} \in E\left(F_{X}^{\mathrm{CR}} / \rho\right)$. Since $\rho$ is a congruence then $(p w q, p v q) \in \rho$ for all $p, q \in\left(F_{X}^{\mathbf{C R}}\right)^{1}$, so $\rho \subseteq \tau$. Similarly $\rho^{\max } \subseteq \tau$ since $\rho^{\max }$ has kernel ker $\rho$. We next show that $\operatorname{ker} \tau^{T}=\operatorname{ker} \rho$.

Suppose $(w, v) \in \tau$ and $w \in \operatorname{ker} \rho$. We consider three cases.

Case 1. Let $w \mathscr{D} v$ in $F_{X}^{\text {CR }}$. With $p=1=q$ in (ii) it follows that $v w^{-1} \in \operatorname{ker} \rho$. Since $w \in \operatorname{ker} \rho$ then $w \rho^{\#}$ is an idempotent, so $\left(v w^{-1}\right) \rho^{\#}=$ ( $v w) \rho^{\#}$ is an idempotent. Also with $p=v$ and $q=w$ in (ii) we get $v v w(v w w)^{-1} \in \operatorname{ker} \rho$. Since $w^{2} \rho^{\#}=w \rho^{\#}$ and $(v w)^{2} \rho^{\#}=(v w) \rho^{\#}$ then $\left(v^{2} w\right) \rho^{\#}=\left(v v w(v w w)^{-1}\right) \rho^{\#}$ is an idempotent. Notice that by Theorem 2.1 $v w, v^{2} w$ and $v^{0}$ are $\mathscr{R}$-related in $F_{X}^{\mathrm{CR}}$. It follows that $(v w) \rho^{\#},\left(v^{2} w\right) \rho^{\#}$ and $v^{0} \rho^{\#}$ are $\mathscr{R}$-related idempotents. Hence $\left(v w v^{0}\right) \rho^{\#}=v^{0} \rho^{\#}$ so $v w v=$ $v w v^{0} v \rho v^{0} v=v$ and likewise $v^{2} w v \rho v$. But then $v \rho v^{2} w v \rho v^{2}$, so $v \in \operatorname{ker} \rho$.

Case 2. Suppose $c(w) \supsetneqq c(v)$. We may assume $c(w)=\left\{x_{1}, \ldots, x_{i}\right\}$ and $c(v)=\left\{x_{1}, \ldots, x_{j}\right\}$ where $j<i$. Let $\phi$ be an endomorphism of $F_{X}^{\text {CR }}$ such that $x_{h} \phi=x_{h}$ if $h \leq j$ and $x_{h} \phi=x_{j}$ if $h>j$. Then $v \phi=v$ and since $\phi$ maps $\operatorname{ker} \rho$ into $\operatorname{ker} \rho$ then $w \phi \in \operatorname{ker} \rho$. Since $\tau$ is fully invariant then $(w \phi, v) \in \tau$. Furthermore $c(w \phi)=c(v)$ so by Theorem $2.1 w \phi \mathscr{D} v$. Thus by Case $1 v \in \operatorname{ker} \rho$.

Case 3. Suppose $c(w) \nsupseteq c(v)$. By (iii) $w^{0} v^{0} \in \operatorname{ker} \rho$. Since $w \in \operatorname{ker} \rho$ then $\left(w, w^{0}\right) \in \rho$ so $\left(w v^{0}, w^{0} v^{0}\right) \in \rho$ and hence $w v^{0} \in \operatorname{ker} \rho$. We have $w v^{0} \tau v v^{0}=v$ since $\tau$ is a right compatible relation. Since $c\left(w v^{0}\right) \supseteq c(v)$ and $v \tau w v^{0} \in \operatorname{ker} \rho$ then by Cases 1 and 2 it follows that $v \in \operatorname{ker} \rho$.

We have seen that if $(w, v) \in \tau$ and $w \in \operatorname{ker} \rho$ then $v \in \operatorname{ker} \rho$. It follows that if $(w, v) \in \tau^{T}$ and $w \in \operatorname{ker} \rho$ then $v \in \operatorname{ker} \rho$. Hence if $v \in$ $\operatorname{ker} \tau^{T}$ then $\left(v^{0}, v\right) \in \tau^{T}$ and since $v^{0} \in \operatorname{ker} \rho$ then $v \in \operatorname{ker} \rho$. Thus $\operatorname{ker} \tau^{T}$ $\subseteq \operatorname{ker} \rho$. But since $\rho \subseteq \tau \subseteq \tau^{T}$ then $\operatorname{ker} \rho \subseteq \operatorname{ker} \tau^{T}$. So $\operatorname{ker} \rho=\operatorname{ker} \tau^{T}$.

We have shown that $\rho^{\max } \subseteq \tau \subseteq \tau^{T}$. But since $\operatorname{ker} \rho=\operatorname{ker} \tau^{T}$ then $\tau^{T} \subseteq \rho^{\max }$. So $\rho^{\max }=\tau$ and the Theorem is proved.

COROLlaRY 4.4. Let $\rho$ and $\theta$ be fully invariant congruences on $F_{X}^{\mathbf{C R}}$. If $\operatorname{ker} \rho \subseteq \operatorname{ker} \theta$ then $\rho^{\max } \subseteq \theta^{\max }$. 
Proof. Define $\tau$ as in the last proof. Define $\tau_{\theta}$ as for $\tau$ but with $\operatorname{ker} \theta$ in place of $\operatorname{ker} \rho$. It follows easily that $\tau \subseteq \tau_{\theta}$. We saw that $\rho^{\max }=\tau$, so $\theta^{\max }=\tau_{\theta}$; hence the result.

Theorem 3.2 and the results of this section together give the following.

TheOREM 4.5. If $\rho$ is a fully invariant congruence on $F_{X}^{\mathrm{CR}}$ then $\rho_{\min }$, $\rho^{\min }, \rho_{\max }$ and $\rho^{\max }$ are fully invariant congruences on $F_{X}^{\mathbf{C R}}$. Moreover, if $\rho$ and $\boldsymbol{\theta}$ are fully invariant congruences on $F_{X}^{\mathbf{C R}}$ then

$$
\begin{gathered}
\operatorname{tr} \rho \subseteq \operatorname{tr} \theta \text { implies } \rho_{\min } \subseteq \theta_{\min } \text { and } \rho_{\max } \subseteq \theta_{\max }, \\
\operatorname{ker} \rho \subseteq \operatorname{ker} \theta \text { implies } \rho^{\min } \subseteq \theta^{\min } \text { and } \rho^{\max } \subseteq \theta^{\max } .
\end{gathered}
$$

5. Sublattices of the lattice of all completely regular semigroup varieties. The lattice of fully invariant congruences on $F_{X}^{\mathbf{C R}}$ is denoted by $C\left(F_{X}^{\mathbf{C R}}\right)$. Let $\rho$ be a fully invariant congruence on $F_{X}^{\mathbf{C R}}$. Then

and

$$
\bar{K}_{\rho}=\left\{\theta \in C\left(F_{X}^{\mathbf{C R}}\right) ; \operatorname{ker} \rho=\operatorname{ker} \theta\right\}=K_{\rho} \cap C\left(F_{X}^{\mathbf{C R}}\right)
$$

$$
\bar{T}_{\rho}=\left\{\theta \in C\left(F_{X}^{\mathbf{C R}}\right) ; \operatorname{tr} \rho=\operatorname{tr} \theta\right\}=T_{\rho} \cap C\left(F_{X}^{\mathbf{C R}}\right)
$$

are intervals of $C\left(F_{X}^{\mathbf{C R}}\right)$. Indeed, according to Theorem 3.2,

$$
\bar{K}_{\rho}=\left[\rho^{\min }, \rho^{\max }\right] \text { and } \bar{T}_{\rho}=\left[\rho_{\min }, \rho_{\max }\right] \text { in } C\left(F_{X}^{\mathbf{C R}}\right) .
$$

In the following result we look again at the situation described in Theorem 3.4.

THEOREM 5.1. Let $\rho$ be a fully invariant congruence on $F_{X}^{\mathrm{CR}}$. Then as intervals of $C\left(F_{X}^{\mathbf{C R}}\right)$

$$
\left[\rho_{\min } \cap \rho^{\min }, \rho\right] \cong\left[\rho_{\min }, \rho\right] \times\left[\rho^{\min }, \rho\right]
$$

by the mutually inverse isomorphisms given by

$$
\theta \rightarrow\left(\theta \vee \rho_{\min }, \theta \vee \rho^{\min }\right), \quad(\zeta, \eta) \rightarrow \zeta \cap \eta .
$$

Proof. Let $\theta \in\left[\rho_{\min } \cap \rho^{\min }, \rho\right]$. Since $\theta \subseteq \rho$ then clearly

$$
\theta \vee \rho_{\min } \in\left[\rho_{\min }, \rho\right] \text { and } \theta \vee \rho^{\min } \in\left[\rho^{\min }, \rho\right] \text {. }
$$

By Theorem 3.4 we have

$$
\operatorname{ker} \rho_{\min }=\operatorname{ker}\left(\rho_{\min } \cap \rho^{\min }\right) \subseteq \operatorname{ker} \theta,
$$

so by Theorem $4.5 \rho_{\min } \subseteq\left(\rho_{\min }\right)^{\max } \subseteq \theta^{\max }$. Thus $\theta \subseteq \theta \vee \rho_{\min } \subseteq \theta^{\max }$ and so $\operatorname{ker} \theta=\operatorname{ker}\left(\theta \vee \rho_{\min }\right)$. In a similar way we get $\operatorname{tr} \theta=\operatorname{tr}\left(\theta \vee \rho^{\min }\right)$. 
Hence since

$$
\theta \subseteq\left(\theta \vee \rho_{\min }\right) \cap\left(\theta \vee \rho^{\min }\right) \subseteq \theta \vee \rho_{\min }
$$

then

$$
\operatorname{ker} \theta=\operatorname{ker}\left(\left(\theta \vee \rho_{\min }\right) \cap\left(\theta \vee \rho^{\min }\right)\right)
$$

Similarly

$$
\operatorname{tr} \theta=\operatorname{tr}\left(\left(\theta \vee \rho_{\min }\right) \cap\left(\theta \vee \rho^{\min }\right)\right) .
$$

Consequently, by (4),

$$
\theta=\left(\theta \vee \rho_{\min }\right) \cap\left(\theta \vee \rho^{\min }\right) .
$$

Let us now suppose that $\zeta \in\left[\rho_{\min }, \rho\right], \eta \in\left[\rho^{\min }, \rho\right]$ and $\theta=\zeta \cap \eta$. Then $\theta \in\left[\rho_{\min } \cap \rho^{\min }, \rho\right]$. Since $\eta \subseteq \rho$ then by Theorem $4.5 \eta_{\min } \subseteq \rho_{\min } \subseteq$ $\zeta$. Hence $\eta_{\min } \subseteq \zeta \cap \eta=\theta \subseteq \eta$.

Thus by the first part of the proof we have $\operatorname{tr} \eta=\operatorname{tr} \theta=\operatorname{tr}\left(\theta \vee \rho^{\min }\right)$.

Furthermore, since $\rho^{\min } \subseteq \eta \subseteq \rho$ and $\rho^{\min } \subseteq \theta \vee \rho^{\min } \subseteq \rho$ then

$$
\operatorname{ker} \eta=\operatorname{ker} \rho=\operatorname{ker}\left(\theta \vee \rho^{\min }\right) \text {. }
$$

Hence by (4), $\eta=\theta \vee \rho^{\min }$. Similarly $\zeta=\theta \vee \rho_{\min }$. Thus the mappings described in the Theorem are mutually inverse bijections.

It is easy to see that the mapping given by $\theta \rightarrow\left(\theta \vee \rho_{\min }, \theta \vee \rho^{\min }\right)$ is a complete $\vee$-homomorphism and that since the domain and range of the mapping are complete lattices then the mapping is a lattice isomorphism with the required inverse.

We remark that in a similar fashion we can show that, as intervals of $C\left(F_{X}^{\mathbf{C R}}\right)$,

$$
\left[\rho_{\min } \cap \rho^{\min }, \rho\right] \cong\left[\rho_{\min } \cap \rho^{\min }, \rho_{\min }\right] \times\left[\rho_{\min } \cap \rho^{\min }, \rho^{\min }\right]
$$

by the mutually inverse isomorphisms

$$
\theta \rightarrow\left(\theta \cap \rho_{\min }, \theta \cap \rho^{\min }\right), \quad(\zeta, \eta) \rightarrow \zeta \vee \eta .
$$

Using Theorem 4.5 we can prove the following result. The proof is similar to the proof for Theorem 3.4 along with that of Theorem 5.1.

THEOREM 5.2. Let $\tau$ be a fully invariant congruence on $F_{X}^{\mathrm{CR}}$. Then

$$
\rho_{\max } \vee \rho^{\max }=\left(\rho^{\max }\right)_{\max } \cap\left(\rho_{\max }\right)^{\max } \in T_{\rho^{\max }} \cap K_{\rho_{\max }}
$$

and as intervals of $C\left(F_{X}^{\mathbf{C R}}\right)$,

$$
\left[\rho, \rho_{\max } \vee \rho^{\max }\right] \cong\left[\rho^{\max }, \rho_{\max } \vee \rho^{\max }\right] \times\left[\rho_{\max }, \rho_{\max } \vee \rho^{\max }\right]
$$




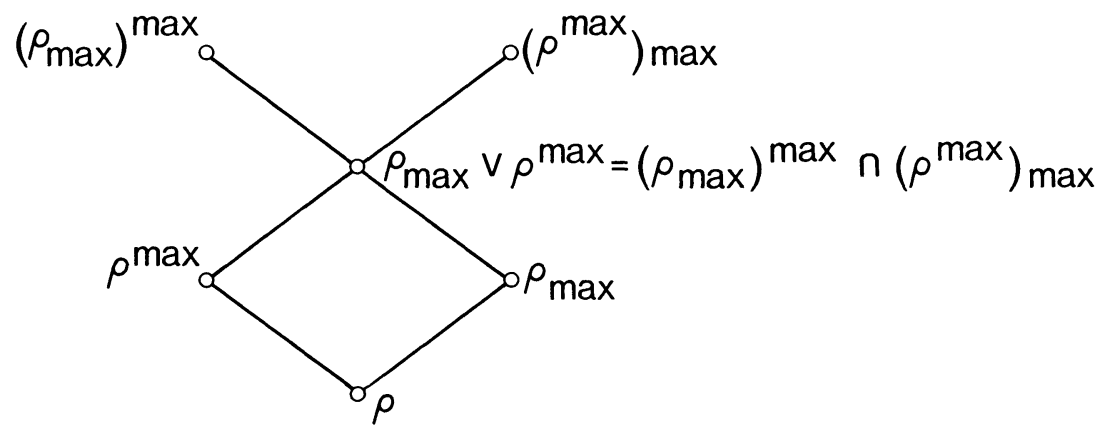

FIGURE 3

by the mutually inverse isomorphisms given by

$$
\theta \rightarrow\left(\theta \vee \rho^{\max }, \theta \vee \rho_{\max }\right), \quad(\zeta, \eta) \rightarrow \zeta \cap \eta .
$$

We remark that in a similar fashion it can be shown that as intervals of $C\left(F_{X}^{\mathbf{C R}}\right)$

$$
\left[\rho, \rho_{\max } \vee \rho^{\max }\right] \cong\left[\rho, \rho^{\max }\right] \times\left[\rho, \rho_{\max }\right]
$$

by the mutually inverse isomorphisms given by

$$
\theta \rightarrow\left(\theta \cap \rho^{\max }, \theta \cap \rho_{\max }\right), \quad(\zeta, \eta) \rightarrow \zeta \vee \eta .
$$

Again, some of the congruences of Figure 3 may coincide.

THEOREM 5.3. Let $\rho$ be a fully invariant congruence on $F_{X}^{\mathbf{C R}}$. Define the congruences $\gamma_{i}, \delta_{i}$ and $\rho_{i}, i \geq 0$, as in Corollary 3.5, and inductively define the congruences $\gamma_{-i}, \delta_{-i}$ and $\rho_{-i}$ as follows:

$$
\gamma_{-i-1}=\left(\delta_{-l}\right)^{\max }, \quad \delta_{-i-1}=\left(\gamma_{-i}\right)_{\max }, \quad \rho_{-i}=\gamma_{-i} \vee \delta_{-i} .
$$

Then $\left\{\gamma_{j}, \delta_{j}, \rho_{j}\right.$; is an integer $\}$ forms a sublattice of $C\left(F_{X}^{\mathbf{C R}}\right)$, where

$$
\rho_{j} \supseteq \rho_{j+1}, \quad \gamma_{j} \supseteq \gamma_{j+1}, \quad \delta_{j} \supseteq \delta_{j+1} \quad \text { and } \quad \gamma_{j} \cap \delta_{j}=\gamma_{j+1} \vee \delta_{j+1}=\rho_{j+1}
$$

for any integer $j$. As intervals of $C\left(F_{X}^{\mathbf{C R}}\right),\left[\rho_{j+1}, \rho_{j}\right] \cong\left[\gamma_{j}, \rho_{j}\right] \times\left[\delta_{j}, \rho_{j}\right]$.

Proof. This follows immediately from Corollary 3.5, Theorem 5.1 and Theorem 5.2.

Recall that $\mathbf{C R}$ is the variety of all completely regular semigroups and $L_{\mathbf{C R}}$ is the lattice of subvarieties of $\mathbf{C R}$. Define $\mathbf{U}_{i}, \mathbf{V}_{i}$ and $\mathbf{W}_{\imath}$ to be, in the notation of Theorem 5.3, the subvarieties of $\mathbf{C R}$ which are determined by the fully invariant congruences $\gamma_{i}, \rho_{i}$ and $\delta_{i}$ respectively for each integer $i$. We obtain a sublattice of $L_{\mathbf{C R}}$ as depicted in Figure 4. However some of 

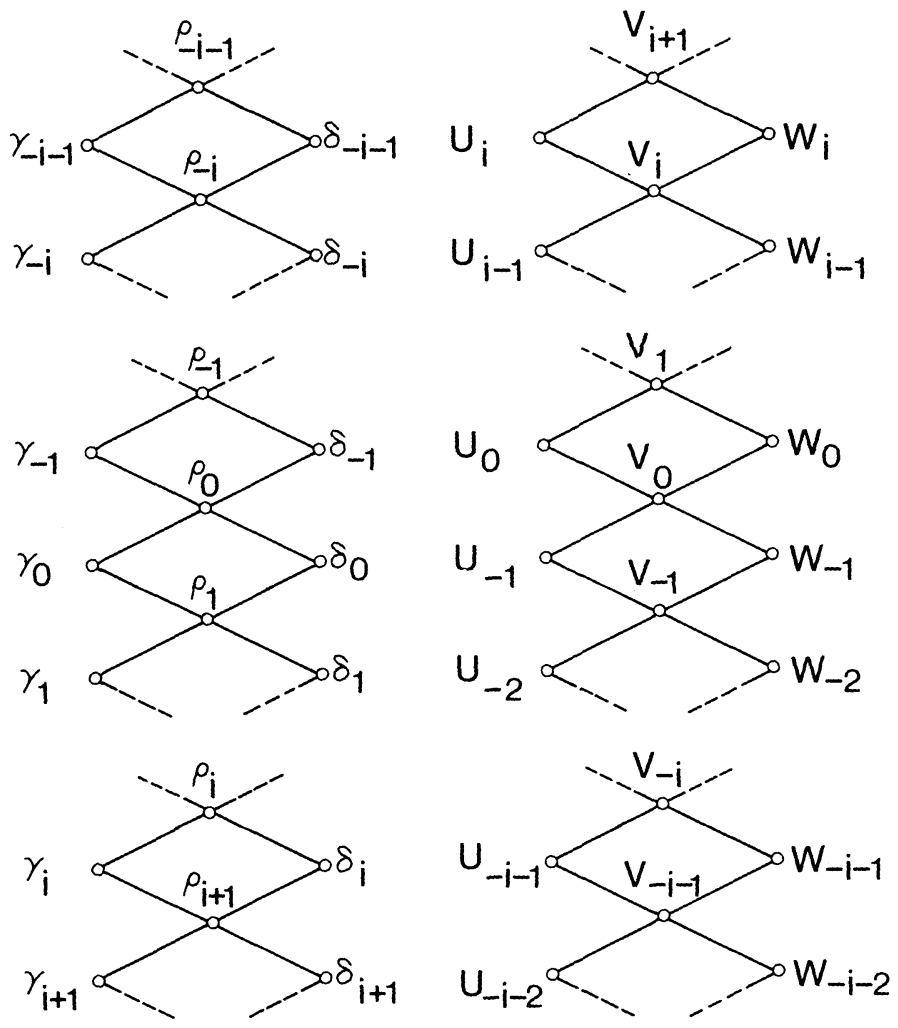

Figure 4

depicted varieties may coincide, in which case the lattice $\left\{\mathbf{U}_{i}, \mathbf{V}_{i}, \mathbf{W}_{i} ; i\right.$ is an integer $\}$ is a homomorphic image of the lattice of Figure 4.

TheOREM 5.4. For $\rho \in C\left(F_{X}^{\mathbf{C R}}\right)$ define the fully invariant congruences $\gamma_{i}, \delta_{i}$ and $\rho_{i}$ for each integer $i$ as in Theorem 5.3. For each $i$ put $\mathbf{U}_{i}=\mathbf{V}_{\gamma_{i}}$, $\mathbf{W}_{i}=\mathbf{V}_{\delta_{i}}$ and $\mathbf{V}_{i}=\mathbf{V}_{\rho_{i}}$. Then in $L_{\mathbf{C R}}$

$$
\left[\mathbf{V}_{i}, \mathbf{V}_{i+1}\right] \cong\left[\mathbf{V}_{i}, \mathbf{U}_{i}\right] \times\left[\mathbf{V}_{i}, \mathbf{W}_{i}\right] \cong\left[\mathbf{U}_{i}, \mathbf{V}_{i+1}\right] \times\left[\mathbf{W}_{i}, \mathbf{V}_{i+1}\right]
$$

by the mutually inverse isomorphisms

$$
\mathbf{V} \rightarrow\left(\mathbf{V} \wedge \mathbf{U}_{i}, \mathbf{V} \wedge \mathbf{W}_{i}\right), \quad(\mathbf{Y}, \mathbf{Z}) \rightarrow \mathbf{Y} \vee \mathbf{Z}
$$

and

$$
\mathbf{V} \rightarrow\left(\mathbf{V} \vee \mathbf{U}_{i}, \mathbf{V} \vee \mathbf{W}_{i}\right), \quad(\mathbf{Y}, \mathbf{Z}) \rightarrow \mathbf{Y} \wedge \mathbf{Z}
$$

respectively. The lattice $\bigcup\left\{\left[\mathbf{W}_{i-1}, \mathbf{U}_{i}\right] ; i\right.$ is an integer $\}$ is modular. If $\mathbf{V} \in$ $\left[\mathbf{V}_{i}, \mathbf{V}_{i+1}\right]$ then $F_{X}^{\mathbf{V}}$ is a subdirect product of $F_{X}^{\mathbf{V}} \wedge \mathbf{w}_{i}$ and $F_{X}^{\mathbf{V}} \wedge \mathbf{U}_{t}$. 
Proof. That the mappings are mutually inverse isomorphisms readily follows from Theorem 5.1, Theorem 5.2 and the remarks following these theorems. The interval $\left[\mathbf{W}_{i-1}, \mathbf{U}_{i}\right]$ of $L_{\mathbf{C R}}$ is anti-isomorphic to the interval $\left[\gamma_{i}, \delta_{i-1}\right]$ of $C\left(F_{X}^{\mathbf{C R}}\right)$. From the definition of $\gamma_{i}$ and $\delta_{i}$ for any $i$, it follows that $\operatorname{tr} \gamma_{i}=\operatorname{tr} \delta_{i-1}$. Hence the interval $\left[\gamma_{i}, \delta_{i-1}\right]$ is embedded in $T_{\gamma_{i}}$, by Theorem 3.2. By [33; Theorem 3.4] $T_{\gamma_{t}}$ is modular. Consequently, the interval $\left[\gamma_{i}, \delta_{i-1}\right]$ of $C\left(F_{X}^{\mathbf{C R}}\right)$ is modular, as is the interval $\left[\mathbf{W}_{i-1}, \mathbf{U}_{i}\right]$ of $L_{\mathbf{C R}}$. Since, by the first part of the theorem,

$$
\left[\mathbf{V}_{i-1}, \mathbf{U}_{i-1}\right] \cup\left[\mathbf{W}_{i-1}, \mathbf{V}_{i}\right] \cong\left[\mathbf{V}_{i-1}, \mathbf{U}_{i-1}\right] \times\left\{\mathbf{V}_{i-1}, \mathbf{W}_{i-1}\right\}
$$

it is now a routine exercise to check that $U\left\{\left[\mathbf{W}_{i-1}, \mathbf{U}_{i}\right] ; i\right.$ an integer $\}$ is a modular lattice.

Let $\mathbf{V} \in\left[\mathbf{V}_{i}, \mathbf{V}_{i+1}\right]$; that is, $\rho_{\mathbf{V}} \in\left[\rho_{i+1}, \rho_{i}\right]$. As in the proof of Theorem 5.1 we have that $\operatorname{ker} \rho_{\mathbf{V}}=\operatorname{ker}\left(\rho_{\mathbf{V}} \vee \gamma_{i}\right)$ and $\operatorname{tr} \rho_{\mathbf{V}}=\operatorname{tr}\left(\rho_{\mathbf{V}} \vee \delta_{i}\right)$. It follows that $\left(\rho_{\mathbf{V}} \vee \gamma_{i}\right) / \rho_{\mathbf{V}}$ is an idempotent pure congruence on $F_{X}^{\mathbf{C R}} / \rho_{\mathbf{v}}=F_{X}^{\mathbf{V}}$ and $\left(\rho_{\mathbf{V}} \vee \delta_{i}\right) / \rho_{\mathbf{V}}$ is an idempotent separating congruence on $F_{X}^{\mathbf{V}}$. Consequently $\left(\rho_{\mathbf{v}} \vee \gamma_{i}\right) / \rho_{\mathbf{v}} \cap\left(\rho_{\mathbf{v}} \vee \delta_{i}\right) / \rho_{\mathbf{v}}$ is the equality on $F_{X}^{\mathbf{v}}$, hence $F_{X}^{\mathbf{V}}$ is a subdirect product of

$$
F_{X}^{\mathbf{V}}\left(\left(\rho_{\mathbf{v}} \vee \delta_{i}\right) / \rho_{\mathbf{v}}\right)^{\#}=F_{X}^{\mathbf{C R}}\left(\rho_{\mathbf{v}} \vee \delta_{i}\right)^{\#}=F_{X}^{\mathbf{V} \wedge \mathbf{w}_{i}}
$$

and

$$
F_{X}^{\mathbf{V}}\left(\left(\rho_{\mathbf{v}} \vee \gamma_{i}\right) / \rho_{\mathbf{v}}\right)^{\#}=F_{X}^{\mathbf{V} \wedge \mathbf{U}_{\iota}}
$$

The first part of the following corollary has been proved by Hall and Jones [15; Corollary 5.7] and Rasin [32; proof of proposition 1].

Corollary 5.5. Let $\mathbf{B}, \mathbf{R B}$ and $\mathbf{C S}$ be the varieties of bands, rectangular bands and completely simple semigroups respectively. Then $[\mathbf{R B}, \mathbf{B} \vee \mathbf{C S}] \cong[\mathbf{R B}, \mathbf{B}] \times[\mathbf{R B}, \mathbf{C S}]$. Furthermore, $F_{X}^{\mathbf{B} \vee \mathbf{C S}}$ is a subdirect product of the free band $F_{X}^{\mathbf{B}}$ and the free completely simple semigroup $F_{X}^{\mathbf{C S}}$.

Proof. Let $\rho=\rho_{\mathbf{R B}}$. Then $\rho_{\text {min }}=\rho_{\mathbf{C S}}, \rho^{\min }=\rho_{\mathbf{B}}$ and the result follows from the Theorem.

The first part of the next corollary is due to Petrich [24] and [25; Theorem 3.3]. Let $L_{\mathbf{V}}$ denote the lattice of subvarieties of a variety $\mathbf{V}$.

Corollary 5.6. Let B, G and OBG be the varieties of bands, groups and orthodox bands of groups respectively. Then $L_{\mathbf{O B G}} \cong L_{\mathbf{B}} \times L_{\mathbf{G}}$. Furthermore $F_{X}^{\mathbf{O B G}}$ is a subdirect product of the free band $F_{X}^{\mathbf{B}}$ and the free group $F_{X}^{\mathbf{G}}$. 
Proof. Let $\rho$ be the universal relation on $F_{X}^{\mathrm{CR}}$. Then $\rho_{\min }=\rho_{\mathrm{G}}$ and $\rho^{\text {min }}=\rho_{\mathbf{B}}$. Let BG and $\mathbf{O}$ be the varieties of bands of groups and of orthodox completely regular semigroups respectively. It is fairly easy to see that $\left(\rho_{\mathbf{B}}\right)_{\min }=\rho_{\mathbf{B G}}$ and by $\left[8\right.$; Theorem 2.4] that $\left(\rho_{\mathbf{G}}\right)^{\min }=\rho_{\mathbf{0}}$. Hence by Theorem 5.3

$$
\mathbf{O B G}=\mathbf{O} \wedge \mathbf{B G}=\mathbf{B} \vee \mathbf{G} .
$$

We note that the result $\mathbf{O B G}=\mathbf{B} \vee \mathbf{G}$ is indirectly (and not so easily) proved in [25; Proposition 3.2]. The Corollary now follows from Theorem 5.4 .

We remark that the above corollaries are only samples of what we can achieve using the techniques developed in this paper. These techniques may facilitate the proving of various results in the existing literature.

In the following the aim is to show that for any fully invariant congruence $\rho$ on $F_{X}^{\text {CR }}$ that is not the equality relation, the lattice $\left\{\gamma_{i}, \delta_{i}, \rho_{i} ; i\right.$ an integer $\}$ of Theorem 5.3 is infinite.

LEMMA 5.7. Let $\rho$ be a congruence on a completely regular semigroup $S$.

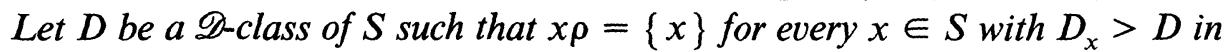
$S / \mathscr{D}$. Then $x\left(\rho^{\min }\right)_{\min }=\{x\}$ for every $x \in S$ with $D_{x} \geq D$ in $S / \mathscr{D}$.

Proof. If $x \in \operatorname{ker} \rho$ then $\left(x, x x^{-1}\right) \in \rho$, so $x \in \operatorname{ker}(\rho \cap \mathscr{D})$. Thus, since $\rho \cap \mathscr{D}$ is a congruence on $S$ it follows by Theorem 3.2 that $\rho^{\min } \subseteq$ $\rho \cap \mathscr{D} \subseteq \mathscr{D}$.

The restriction $\rho \mid D$ of $\rho$ to $D$ is a congruence on the completely simple semigroup $D$. It is easy to see that $(\rho \mid D)^{\min }$ is an idempotent separating congruence on $D$. Since $x \rho^{\min }=\{x\}$ for every $x \in S$ with $D_{x}>D$ in $S / \mathscr{D}$, since $\rho^{\min } \subseteq \mathscr{D}$ and since $(\rho \mid D)^{\min } \subseteq \rho^{\min } \mid D$ we see that $\rho^{\min } \mid(S \backslash D) \cup(\rho \mid D)^{\min }$ is a congruence on $S$. Also since $\rho^{\min } \subseteq \mathscr{D}$ then

$$
\operatorname{ker}(\rho \mid D)^{\min }=\operatorname{ker} \rho\left|D=\operatorname{ker} \rho^{\min }\right| D
$$

so

$$
\operatorname{ker}\left(\rho^{\min } \mid(S \backslash D) \cup(\rho \mid D)^{\min }\right)=\operatorname{ker} \rho^{\min } .
$$

Consequently $\rho^{\min }=\rho^{\min } \mid(S \backslash D) \cup(\rho \mid D)^{\min }$, and $\rho^{\min }$ separates the idempotents of $D$. Let $\iota_{D}$ be the equality on $D$. Then $\rho^{\min } \mid(S \backslash D) \cup \iota_{D}$ is a congruence on $S$ with trace $\operatorname{tr} \rho^{\min }$. Thus $\left(\rho^{\min }\right)_{\min } \subseteq \rho^{\min } \mid(S \backslash D) \cup \iota_{D}$. Hence $\left(\rho^{\min }\right)_{\min } \mid D=\iota_{D}$ and the result follows.

Let $F_{n}{ }^{\mathbf{C R}}$ denote the free completely regular semigroup on an $n$ element set and $\mathbf{F}_{n}$ be the variety of completely regular semigroups generated by $F_{n}^{\mathbf{C R}}$. 
THEOREM 5.8. Let $\rho \in C\left(F_{X}^{\mathbf{C R}}\right)$ be different from the equality relation. Then, in the notation of Theorem $5.4, \mathbf{U}_{2 n-1} \geq \mathbf{F}_{n}$ for every integer $n \geq 1$.

Proof. Let $Y$ be a finite subset of $X$. By Theorem 2.1 the set $D_{Y}$ of all $w \in F_{X}^{\text {CR }}$ with content $c(w)=Y$ is a $\mathscr{D}$-class. Let $C_{n}=\bigcup_{|Y| \leq n} D_{Y}$ for each integer $n \geq 1$, where $C_{0}=\square$. In the notation of Theorem 5.3 we have $\gamma_{2(n+1)-1}=\gamma_{2 n+1}=\left(\gamma_{2 n-1}{ }^{\mathrm{min}}\right)_{\min }$. Hence, by a simple induction argument utilizing Lemma 5.7 we get $w \gamma_{2 n-1}=\{w\}$ for all $w \in C_{n}$ and each integer $n \geq 0$. For $n \geq 1$, any subsemigroup of $F_{X}^{\mathbf{C R}}$ generated by $n$ elements of $X$ is isomorphic to $F_{n}{ }^{\mathbf{C R}}$ and is contained in $C_{n}$. It follows that $F_{n}{ }^{\mathbf{C R}}$ is embedded in $F_{X}^{\mathbf{C R}} / \gamma_{2 n-1}=F_{X}^{\mathbf{U}_{n-1}}$ for each integer $n \geq 1$. Since $\mathbf{U}_{2 n-1}$ is the variety generated by $F_{X}^{\mathbf{U}_{2 n-1}}$ we conclude that $\mathbf{F}_{n} \leq \mathbf{U}_{2 n-1}$.

COROLlaRY 5.9. In the notation of Theorem 5.4, if $\rho \in C\left(F_{X}^{\mathrm{CR}}\right)$ then

$$
\bigvee_{n \geq 1} \mathbf{U}_{n}=\bigvee_{n \geq 1} \mathbf{V}_{n}=\bigvee_{n \geq 1} \mathbf{W}_{n}=\bigvee_{n \geq 1} \mathbf{F}_{n}=\mathbf{C R}
$$

The following theorem tells us that if $\rho \in C\left(F_{X}^{\mathbf{C R}}\right)$ is different from the equality relation then $\rho_{\min } \varsubsetneqq \rho$ or $\rho^{\min } \varsubsetneqq \rho$.

THeOREM 5.10. Let $\rho \in C\left(F_{X}^{\mathbf{C R}}\right)$ and $\rho_{\min }=\rho=\rho^{\min }$ then $\rho$ is the equality relation.

Proof. If $\rho_{\min }=\rho=\rho^{\min }$ then in the notation of Theorem 5.3, $\rho=$ $\left(\rho^{\min }\right)_{\min }=\gamma_{1}$. By induction, $\rho=\gamma_{2 n-1}$ for every integer $n \geq 1$. Hence by Corollary 5.9, $\rho=\bigcap_{n \geq 1} \gamma_{2 n-1}$ is the equality relation.

LEMMA 5.11. For any infinite set $Z$ the equality relation is the only idempotent pure and the only idempotent separating congruence on $F_{Z}^{\mathbf{C R}}$.

Proof. Let $v, w \in F_{Z}^{\mathbf{C R}}$ and $w \neq v$ in $F_{Z}^{\mathbf{C R}}$. Let $c(v) \cup c(w)=$ $\left\{x_{2}, \ldots, x_{n-1}\right\} \subseteq Z$ and select distinct elements $x_{1}, x_{n} \in Z \backslash(c(v) \cup$ $c(w))$. Put $f=\left(x_{1} \cdots x_{n}\right)^{0}$. From Theorem 2.1 then $w f$ and $v f$ are not $\mathscr{R}$-related in $F_{Z}^{\mathbf{C R}}$. Hence the congruence on $F_{Z}^{\mathbf{C R}}$ that is generated by $(v, w)$ is not idempotent separating. Furthermore since $x_{1}, x_{n} \notin c(v) \cup$ $c(w)$ then by [38; Theorem 4.1 and Definitions 3.2, 3.5 and $(\theta 7)$ ] it follows that $f v f$ and $f w f$ are distinct; they are $\mathscr{H}$-related by Theorem 2.1. Thus the congruence generated by $(v, w)$ is not idempotent pure.

We note that with more reliance on technical detail from [38] it can be shown that a free completely regular semigroup on any set has no non-equality idempotent pure congruence. 
THEOREM 5.12. For any $\rho \in C\left(F_{X}^{\mathbf{C R}}\right)$ that is different from the equality relation, the chain $\left\{\mathbf{U}_{2 n-1} ; n \geq 1\right\}$ of Theorem 5.4 is strictly increasing.

Proof. Suppose the chain is not strictly increasing and $n$ is the smallest positive integer for which $\mathbf{U}_{2 n-1}=\mathbf{U}_{2 n+1}$. Then $\gamma_{2 n-1}=\gamma_{2 n+1}$, so $\gamma_{2 n-1}=\left(\gamma_{2 n-1}\right)_{\min }=\left(\gamma_{2 n-1}\right)^{\min }$ and by Theorem $5.10 \gamma_{2 n-1}$ is the equality relation. Thus $\gamma_{2 n-1}<\delta_{2 n-2}$ or $\gamma_{2 n-1}=\delta_{2 n-2}$ and $\delta_{2 n-2}<\gamma_{2 n-3}$. In the former case $\delta_{2 n-2}$ is a proper idempotent separating congruence on $F_{X}^{\text {CR }}$ and in the latter case $\gamma_{2 n-3}$ is a proper idempotent pure congruence on $F_{X}^{\text {CR }}$. By Lemma 5.11 this is impossible.

In the following we shall show that the chain of varieties $\left\{\mathbf{F}_{n} ; n \geq 1\right\}$ is also strictly increasing. We suppose $x_{1}, \ldots, x_{n+2} \in X$, and define elements $u_{n}, v_{n} \in F_{X}^{\mathbf{C R}}$ inductively for integers $n \geq 1$, as follows:

$$
\begin{gathered}
u_{0}=x_{1}, \quad v_{0}=x_{2} \quad \text { and, for } n \geq 0, \\
u_{n+1}=u_{n}^{0} v_{n}^{0} x_{n+3}^{0} u_{n}^{0} v_{n}^{0}, \quad v_{n+1}=\left(u_{n}^{0} v_{n}^{0}\right)^{0} x_{n+3}^{0}\left(u_{n}^{0} v_{n}^{0}\right)^{0} .
\end{gathered}
$$

Clearly $c\left(u_{n}\right)=c\left(v_{n}\right)=\left\{x_{1}, \ldots, x_{n+2}\right\}$ for $n \geq 1$.

LEMMA 5.13. Let $\rho$ be the universal relation on $F_{X}^{\mathbf{C R}}$. In the notation of Theorem 5.4, $\mathbf{F}_{n+1} \leq \mathbf{U}_{2 n}$ for all integers $n \geq 0$. For any $n \geq 0$ let the identity $u_{n}=v_{n}$ be defined by (9). Then $u_{n}^{0}=v_{n}^{0}$ is satisfied in $\mathbf{U}_{2 n}$ but not in $\mathbf{F}_{n+2}$.

Proof. Since $\rho$ is universal then $\gamma_{0}=\rho_{\min }$ is the least group congruence on $F_{X}^{\mathbf{C R}}$. Then $F_{X}^{\mathbf{C R}} / \gamma_{0}$ is a free group. Since any $x \in X$ generates the free cyclic group as a subsemigroup of $F_{X}^{\mathbf{C R}}$ [2; Proposition 3.3] then $w \gamma_{0}=\{w\}$ for any $w \in F_{X}^{\text {CR }}$ where $|c(w)|=1$; that is, for $w \in C_{1}$. We may continue as in the proof of Theorem 5.8 to get $\mathbf{F}_{n+1} \leq \mathbf{U}_{2 n}$ for all $n \geq 1$.

The identity $u_{0}^{0}=v_{0}^{0}$ determines the variety of all groups, namely $\mathbf{U}_{0}$. By $[2 ; \S 6]$ the identity $u_{0}^{0} v_{0}^{0}=\left(u_{0}^{0} v_{0}^{0}\right)^{0}$ is not satisfied in $F_{2}^{\mathbf{C R}}$, so $u_{0}^{0}=v_{0}^{0}$ is not satisfied in $\mathbf{F}_{2}$. Suppose that $u_{n}^{0}=v_{n}^{0}$ is satisfied in $\mathbf{U}_{2 n}$ and that $u_{n}^{0} v_{n}^{0}=\left(u_{n}^{0} v_{n}^{0}\right)^{0}$ is not satisfied in $\mathbf{F}_{n+2}$. We proceed by induction. Since $\left(u_{n}^{0}, v_{n}^{0}\right) \in \gamma_{2 n}$ then $\left(u_{n}^{0} v_{n}^{0}, u_{n}^{0}\right) \in \gamma_{2 n}$ so $u_{n}^{0} v_{n}^{0} \in \operatorname{ker} \delta_{2 n+1}$. Thus $\left(u_{n}^{0} v_{n}^{0},\left(u_{n}^{0} v_{n}^{0}\right)^{0}\right) \in \delta_{2 n+1}$ and since $\delta_{2 n+1}$ is a congruence then $\left(u_{n+1}, v_{n+1}\right)$ $\in \delta_{2 n+1}$. Hence by [16; Theorem I.5.6],

$$
u_{n+1} \gamma_{2 n+2}^{\#}\left(\delta_{2 n+1} / \gamma_{2 n+2}\right)^{\#}=v_{n+1} \gamma_{2 n+2}^{\#}\left(\delta_{2 n+1} / \gamma_{2 n+2}\right)^{\#} \text {. }
$$

It follows, since $\delta_{2 n+1} / \gamma_{2 n+2}$ is idempotent separating on $F_{X}^{\mathbf{C R}} / \gamma_{2 n+2}=$ $F_{X}^{\mathbf{U}_{2 n+2}}$, that $u_{n+1} \gamma_{2 n+2}^{\#} \mathscr{H}_{n+1} \gamma_{2 n+2}^{\#}$ so $\left(u_{n+1}^{0}, v_{n+1}^{0}\right) \in \gamma_{2 n+2}$. Hence $u_{n+1}^{0}=$ $v_{n+1}^{0}$ in $\mathbf{U}_{2 n+2}$. The left indicators of $u_{n+1}^{0}$ and $v_{n+1}^{0}$ are $u_{n}^{0} v_{n}^{0} x_{n+3}$ and 
$\left(u_{n}^{0} v_{n}^{0}\right)^{0} x_{n+3}$ respectively. Since we may regard $F_{n+2}^{\mathbf{C R}}$ and $F_{n+3}^{\mathbf{C R}}$ as being the subsemigroups of $F_{X}^{\mathbf{C R}}$ generated respectively by $\left\{x_{1}, \ldots, x_{n+2}\right\}$ and $\left\{x_{1}, \ldots, x_{n+3}\right\}$, then by the induction assumption $u_{n}^{0} v_{n}^{0} \neq\left(u_{n}^{0} v_{n}^{0}\right)^{0}$ in $F_{n+3}^{\mathbf{C R}}$. Hence by Theorem 2.1, $u_{n+1}^{0} \neq v_{n+1}^{0}$ in $F_{n+3}^{\mathbf{C R}}$. So $u_{n+1}^{0}=v_{n+1}^{0}$ is not an identity in $\mathbf{F}_{n+3}$.

In order to complete the proof we must check that $u_{n+1}^{0} v_{n+1}^{0} \neq$ $\left(u_{n+1}^{0} v_{n+1}^{0}\right)^{0}$ in $F_{n+3}^{\mathbf{C R}}$. Let $f=\left(x_{1} x_{2} \cdots x_{n+3}\right)^{0}$. Since both sides of the following are idempotents then by Theorem 2.1

$$
\begin{aligned}
u_{n+1}^{0} & =u_{n}^{0} v_{n}^{0} x_{n+3} f\left(f\left(u_{n}^{0} v_{n}^{0}\right)^{2} x_{n+3} f\right)^{-1} f u_{n}^{0} v_{n}^{0}, \\
v_{n+1}^{0} & =\left(u_{n}^{0} v_{n}^{0}\right)^{0} x_{n+3} f\left(f\left(u_{n}^{0} v_{n}^{0}\right)^{0} x_{n+3} f\right)^{-1} f\left(u_{n}^{0} v_{n}^{0}\right)^{0}, \\
\left(u_{n+1}^{0} v_{n+1}^{0}\right)^{0} & =u_{n}^{0} v_{n}^{0} x_{n+3} f\left(f u_{n}^{0} v_{n}^{0} x_{n+3} f\right)^{-1} f\left(u_{n}^{0} v_{n}^{0}\right)^{0} .
\end{aligned}
$$

Then

$$
\begin{aligned}
u_{n+1}^{0} v_{n+1}^{0}= & u_{n}^{0} v_{n}^{0} x_{n+3} f\left(f\left(u_{n}^{0} v_{n}^{0}\right)^{2} x_{n+3} f\right)^{-1} f u_{n}^{0} v_{n}^{0} x_{n+3} \\
& \cdot f\left(f\left(u_{n}^{0} v_{n}^{0}\right)^{0} x_{n+3} f\right)^{-1} f\left(u_{n}^{0} v_{n}^{0}\right)^{0} .
\end{aligned}
$$

Since $\left(u_{n}^{0} v_{n}^{0}\right)^{0}, u_{n}^{0} v_{n}^{0}$ and $\left(u_{n}^{0} v_{n}^{0}\right)^{2}$ each have content $c(f) \backslash\left\{x_{n+3}\right\}$ and are distinct by the induction assumption then by [38; $(\theta)$ and Corollary 4.2] $f u_{n}^{0} v_{n}^{0} x_{n+3} f, f\left(u_{n}^{0} v_{n}^{0}\right)^{0} x_{n+3} f$ and $f\left(u_{n}^{0} v_{n}^{0}\right)^{2} x_{n+3} f$ are distinct free generators of an $\mathscr{H}$-class of $F_{n+3}^{\mathbf{C R}}$. So $u_{n+1}^{0} v_{n+1}^{0} \neq\left(u_{n+1}^{0} v_{n+1}^{0}\right)^{0}$ in $F_{n+3}^{\mathbf{C R}}$

COROllary 5.14. For integers $n \geq 0$ let $u_{n}$ and $v_{n}$ be given by (9), and let $\left[u_{n}^{0}=v_{n}^{0}\right]$ be the variety of completely regular semigroups determined by the identity $u_{n}^{0}=v_{n}^{0}$. Then $\mathbf{F}_{n+1} \leq\left[u_{n}^{0}=v_{n}^{0}\right] \cap \mathbf{F}_{n+2} \lesseqgtr \mathbf{F}_{n+2}$.

Remarks. Suppose $\rho$ is the universal congruence on $F_{X}^{\text {CR }}$. In the notation of Theorem 5.4 then $\mathbf{U}_{0}=\mathbf{G}$ is the variety of all groups, $\mathbf{W}_{0}=\mathbf{B}$ is the variety of all bands, $\mathbf{V}_{1}$ is the variety of all orthocryptogroups (orthodox bands of groups), $\mathbf{U}_{1}=\mathbf{B G}$ is the variety of all cryptogroups (bands of groups) and $\mathbf{W}_{1}=\mathbf{0}$ is the variety of all orthogroups (orthodox completely regular semigroups). The congruences $\left(\rho_{\mathbf{0}}\right)_{\min }$ and $\left(\rho_{\mathbf{B G}}\right)^{\min }$ respectively induce $U_{2}$ and $W_{2}$. According to Theorem 4.1, $U_{2}$ is determined by all identities of the form $w w^{-1}=v v^{-1}$ which hold in 0 and $\mathbf{W}_{2}$ is determined by all identities of the form $u^{2}=u$ which holds in BG. Since $\mathbf{0} \vee \mathbf{B G}=\mathbf{U}_{2} \wedge \mathbf{W}_{2}$ we have that $\mathbf{0} \vee \mathbf{B G}$ is determined by all identities of the form $w w^{-1}=v v^{-1}$ which hold in 0 and $u^{2}=u$ which hold in BG. Furthermore by Theorem 5.4 the free object $F_{X}^{\mathbf{O} \vee \mathbf{B G}}$ is a subdirect product 
of the free orthogroup $F_{X}^{\mathbf{O}}$ and the free cryptogroup $F_{X}^{\mathbf{B G}}$. These observations partially answer the question of Petrich [27; Problem 2].

Since $\left(\delta_{1} / \gamma_{2}\right)^{\#:} F_{X}^{\mathbf{U}_{2}} \rightarrow F_{X}^{\mathbf{W}_{1}}=F_{X}^{\mathbf{O}}$ is idempotent separating and since $F_{X}^{\mathbf{O}}$ is orthodox it follows that the core of $F_{X}^{\mathbf{L}_{2}}$, that is, the idempotent generated part of $F_{X}^{\mathbf{U}_{2}}$, is a cryptogroup. Using Lallement's Lemma one easily shows that every homomorphic image of $F_{X}^{\mathbf{U}_{2}}$ has the same property. Hence the core of every $S \in \mathrm{U}_{2}$ is a cryptogroup. Conversely, if the core of a completely regular semigroup $S$ is a cryptogroup and if $\mu_{S}$ is the greatest idempotent separating congruence on $S$ then using Hall's characterization of $\mu_{S}$ [14] we easily see that $S / \mu_{S} \in \mathbf{O}$. Since $\operatorname{tr} \mu_{S}$ is the equality relation on $E(S)$ it follows that $S$ satisfies all identities of the form $w w^{-1}=v v^{-1}$ that hold in $\mathbf{O}$. Thus $S \in \mathbf{U}_{2}$. We conclude that $S \in \mathbf{U}_{2}$ if and only if the core of $S$ is a cryptogroup. In particular, by Theorem 5.13 the core of $F_{2}{ }^{\text {CR }}$ is a cryptogroup.

By the dual of Theorem 2.1(iii) $\left((p v p)^{0}(p u p)^{0}\right)^{0}$ and $\left((p u p)^{0}(p v p)^{0}(p u p)^{0}\right)^{0}$ are $\mathscr{L}$-related idempotents of $F_{2}^{\mathbf{C R}}$ for all $u, v, p \in$ $F_{2}^{\text {CR }}$. In fact since $|c(w)| \leq 2$ for all $w \in F_{2}^{\text {CR }}$ we get that

$$
\left((p w p)^{0}\left((p v p)^{0}(p u p)^{0}\right)^{0}\right)^{0} \text { and }\left((p w p)^{0}\left((p u p)^{0}(p v p)^{0}(p u p)^{0}\right)^{0}\right)^{0}
$$

are $\mathscr{L}$-related idempotents of $F_{2}^{\text {CR }}$. Let

$$
\begin{aligned}
& a=\left(\left(x_{4} x_{3} x_{4}\right)^{0}\left(\left(x_{4} x_{1} x_{4}\right)^{0}\left(x_{4} x_{2} x_{4}\right)^{0}\left(x_{4} x_{1} x_{4}\right)^{0}\right)^{0}\right)^{0}, \\
& b=\left(\left(x_{4} x_{3} x_{4}\right)^{0}\left(\left(x_{4} x_{2} x_{4}\right)^{0}\left(x_{4} x_{1} x_{4}\right)^{0}\right)^{0}\right)^{0} .
\end{aligned}
$$

Then $a b=a$ is an identity satisfied in $\mathbf{F}_{2}$. By Green and Rees [13] (see [16; Lemma 4.6]) Theorem 2.1 holds for free bands with the free unary semigroup $F_{X}^{\mathbf{U}}$ replaced by the free semigroup $F_{X}$ on $X$ and with $\lambda$ replaced by the congruence generated by $\left\{\left(w, w^{2}\right) ; w \in F_{X}\right\}$. If $a b=a$ in $F_{X}^{\mathbf{B}}$ then by this version of Theorem $2.1 R(a b)=R(a)$ in $F_{X}^{\mathbf{B}}$ and again by Theorem 2.1 $L(R(a b))=L(R(a))$. But $L(R(a b))=x_{3} x_{4}\left(x_{4} x_{2} x_{4}\right)^{0} x_{4} x_{1}$ while $L(R(a))=x_{3} x_{4}\left(x_{4} x_{1} x_{4}\right)^{0} x_{4} x_{2}$. Theorem 2.1 demands that $x_{1}=x_{2}$ so we conclude that $a b=a$ is an identity not satisfied in B. Since $\mathbf{B}<\mathbf{O}<\mathbf{U}_{2}$ we then have that $\mathbf{F}_{2} \lesseqgtr \mathbf{U}_{2}$.

Since $F_{1}$ is the variety generated by the free cyclic group then $F_{1}$ is the variety of all abelian groups. $\mathbf{U}_{0}$ is the variety of all groups so $\mathbf{F}_{1} \supsetneqq \mathbf{U}_{0}$.

Let $F_{n}$ CR be freely generated by a set $Z$ of $n$ elements Let $D_{n}$ denote the $\mathscr{D}$-class of elements of content $Z$. Then $D_{n}$ is a completely simple subsemigroup and ideal of $F_{n}^{\text {CR }}$. Define a relation $\tau$ on $F_{n}{ }^{\mathbf{C R}}$ such that $\tau$ is the equality relation on $F_{n}^{\mathrm{CR}} \backslash D_{n}$ and $\tau$ is the $\mathscr{H}$-relation on $D_{n}$. Since $\mathscr{H}$ is 
a congruence on $D_{n}$ then we see by Theorem 2.1 that $\tau$ is a congruence on $F_{n}^{\mathbf{C R}}$. In fact $\tau$ is idempotent separating. We see that $F_{n}^{\mathbf{C R}} / \tau$ has an ideal that is an $I \times I$ rectangular band where, by Theorem $2.1, I$ is a countable set. Hence since varieties are closed under the taking of homomorphic images and the taking of subsemigroups we see that $\mathbf{F}_{n}$ contains the variety of all rectangular bands $\mathbf{R B}$.

Note also that $\mathbf{F}_{n}$ contains the variety of all groups since by $[2 ; \S 6]$ $F_{n}^{\text {CR }}$ contains subgroups that are free of countably infinite rank. Thus RB $\vee \mathbf{G} \leq \mathbf{F}_{2} \lesseqgtr \mathbf{U}_{2}$. We summarise these remarks for $\mathbf{U}_{2}$.

THEOREM 5.15. Let $\rho$ be a universal congruence on $F_{X}^{\mathrm{CR}}$. In the notation of Theorem 5.4, $S \in \mathbf{U}_{2}$ if and only if $S$ is a completely regular semigroup whose core is a cryptogroup. Furthermore $\mathbf{R B} \vee \mathbf{G} \leq \mathbf{F}_{2} \lesseqgtr \mathbf{U}_{2}$.

The following theorem is an easy corollary of the results of the paper and the remarks.

THEOREM 5.16. For any non-empty set $Z, Z$ is infinite if and only if the only idempotent separating congruence on $F_{Z}^{\mathbf{C R}}$ is the equality relation.

Proof. Necessity is by Lemma 5.11. Sufficiency follows from the construction of the congruence $\tau$ on $F_{n}^{\mathbf{C R}}$ in the remarks preceding this Theorem.

\section{REFERENCES}

[1] A. P. Birjukov, Varieties of idempotent semigroups, Algebra i Logika, 9 (1970), 255-273. (in Russian)

[2] A. H . Clifford, The free completely regular semigroup on a set, J. Algebra, 59 (1979), 434-451.

[3] A. H. Clifford and G. B. Preston, The algebraic theory of semigroups, Vol. II, Amer. Math. Soc., Providence, 1967.

[4] R. Feigenbaum, Kernels of regular semigroup homomorphisms, Doctoral dissertation, University of South Carolina, 1975.

[5] R. Feigenbaum, Regular semigroup congruences, Semigroup Forum, 17 (1979), 373-377.

[6] C. F. Fennemore, All varieties of bands, Math. Nachr., 48 (1971); I: 237-252; II; 253-262.

[7] J. A. Gerhard, The lattice of equational classes of idempotent semigroups, J. Algebra, 15 (1970), 195-224.

[8] J. A. Gerhard and M. Petrich, The word problem for orthogroups, Canad. J. Math., 33 (1981), 893-900. 
[9] J. A. Gerhard, Free completely regular semigroups I, J. Algebra, 82 (1983), 135-142.

[10] _ Free completely regular semigroups II, J. Algebra, 82 (1983), 143-156.

[11] G. Gratzer, Universal Algebra, Van Nostrand, Princeton, 1968.

[12] D. G. Green, The lattice of congruences on an inverse semigroup, Pacific J. Math., 57 (1975), 141-152.

[13] J. A. Green and D. Rees, On semigroups in which $x^{r}=x$, Proc. Cambridge Phil. Soc., 48 (1952), 35-40.

[14] T. E. Hall, On regular semigroups, J. Algebra, 24 (1973), 1-24.

[15] T. E. Hall and P. R. Jones, On the lattice of varieties of bands of groups, Pacific J. Math., 91 (1980), 327-337.

[16] J. M. Howie, An Introduction to Semigroup Theory, Academic Press, London/New York, 1976.

[17] P. R. Jones, Universal Aspects of Completely Simple Semigroups, Semigroups (Ed. T. E. Hall, P. R. Jones, G. B. Preston) Academic Press, New York, 1980, 27-46.

[18] _ Completely simple semigroups: free products, free semigroups and varieties, Proc. Royal Soc. Edinburugh, A 88 (1981), 293-313.

[19] _ On the lattice of varieties of completely regular semigroups, J. Austral. Math. Soc. (Series A), 35 (1983), 227-235.

[20] G. Lallement, Congruences et équivalences de Green sur un demi-groupe régulier, C. R. Acad. Sci. Paris, Sér. A, 262 (1966), 613-616.

[21] G. I. Masevickir, On identities in varieties of completely simple semigroups over abelian groups, Contemporary algebra, Leningrad (1978), 81-89 (in Russian).

[22] K. S. S. Nambooripad, Structure of regular semigroups I, Semigroup Forum, 9 (1974), 354-363.

[23] _ Structure of regular semigroups I, Memoirs Amer. Math. Soc., 244, Providence, 1979.

[24] M. Petrich, Varieties of orthodox bands of groups, Pacific J. Math., 58 (1975), 209-217.

[25] __ Certain varieties and quasivarieties of completely regular semigroups, Canad. J. Math., 29 (1977), 1171-1197.

[26] Congruences on inverse semigroups, J. Algebra, 55 (1978), 231-256.

[27] _ On the varieties of completely regular semigroups, Semigroup Forum, 25 (1982), 153-169.

[28] M. Petrich and N. R. Reilly, Varieties of groups and of completely simple semigroups, Bull. Austral. Math. Soc., 23 (1981), 339-359.

[29] _ A network of congruences on an inverse semigroup, Trans. Amer. Math. Soc., 270 (1982), 309-325.

[30] V. V. Rasin, On the varieties of bands of groups, Trans. XV All union algebraic conference, Krasnojarsk (1979), Vol. 2, 123 (in Russian).

[31] _... On the lattice of varieties of completely simple semigroups, Semigroup Forum, 17 (1979), 113-122.

[32] _ On the varieties of Cliffordian semigroups, Semigroup Forum, 23 (1981), 201-220.

[33] N. R. Reilly and H. E. Scheiblich, Congruences on regular semigroups, Pacific J. Math., 23 (1967), 349-360.

[34] H. E. Scheiblich, Kernels of inverse semigroup homomorphisms, J. Austral. Math. Soc., 18 (1974), 289-292.

[35] M. Teissier, Sur les équivalences regulières dans les demi-groupes, C. R. Acad. Sci. Paris, 232 (1951), 1987-1989.

[36] P. G. Trotter, Normal partitions of idempotents of regular semigroups, J. Austral. Math. Soc. (Series A), 26 (1978), 110-114. 
[37] Congruences on regular and completely regular semigroups, J. Austral. Math. Soc., (Series A), 32 (1982), 388-398.

[38] _ Free completely regular semigroups, Glasgow Math. J., 25 (1984), 241-254.

Received December 29, 1983.

Dienst Hogere MeETKunde, RIJKSUNIVERSITEIT TE GENT

KRIJGSLAAN 281

B - 9000 GENT, BELGIUM

AND

UNIVERSITY OF TASMANIA

Hobart, TASMANIA

Australia 


\section{PACIFIC JOURNAL OF MATHEMATICS EDITORS}

\author{
V. S. VARADARAJAN (Managing Editor) \\ University of California \\ Los Angeles, CA 90024 \\ Charles R. DePrima \\ California Institute of Technology \\ Pasadena, CA 91125 \\ R. FINN \\ Stanford University \\ Stanford, CA 94305
}

\author{
HeRmanN FlaschKa \\ University of Arizona \\ Tucson, AZ 85721 \\ Ramesh A. Gangolli \\ University of Washington \\ Seattle, WA 98195 \\ ROBION KIRBY \\ University of California \\ Berkeley, CA 94720
}

C. C. MOORE

University of California

Berkeley, CA 94720

H. SAMELSON

Stanford University

Stanford, CA 94305

HAROLD STARK

University of California, San Diego

La Jolla, CA 92093

ASSOCIATE EDITORS
R. ARENS
E. F. BECKENBACH
B. H. NeUmanN
F. WOLF
K. YosHIDA (1906-1982)

\section{SUPPORTING INSTITUTIONS}

UNIVERSITY OF ARIZONA

UNIVERSITY OF BRITISH COLUMBIA

CALIFORNIA INSTITUTE OF TECHNOLOGY

UNIVERSITY OF CALIFORNIA

MONTANA STATE UNIVERSITY

UNIVERSITY OF NEVADA, RENO

NEW MEXICO STATE UNIVERSITY

OREGON STATE UNIVERSITY
UNIVERSITY OF OREGON

UNIVERSITY OF SOUTHERN CALIFORNIA

STANFORD UNIVERSITY

UNIVERSITY OF HAWAII

UNIVERSITY OF TOKYO

UNIVERSITY OF UTAH

WASHINGTON STATE UNIVERSITY

UNIVERSITY OF WASHINGTON 


\section{Pacific Journal of Mathematics}

Vol. 119, No. $1 \quad$ May, 1985

Maurice Chacron, Nonisotropic unitary spaces and modules with

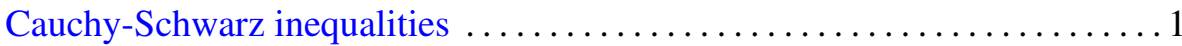

Myriam Dechamps-Gondim, Françoise Piquard and H. Queffélec, On

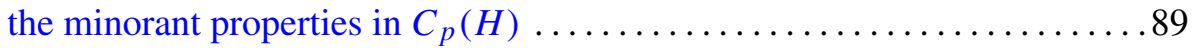

Klaus Floret and V. B. Moscatelli, On bases in strict inductive and projective limits of locally convex spaces .................... 103

Norman Joseph Goldstein, Degenerate secant varieties and a problem on

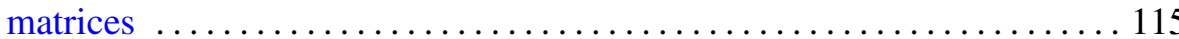

Harold Morris Hastings and Stefan Waner, $G$-bordism with singularities

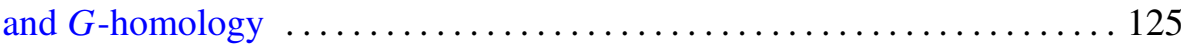

Takesi Isiwata, Clopen realcompactification of a mapping ........... 153

Hisao Kato, Concerning hyperspaces of certain Peano continua and strong

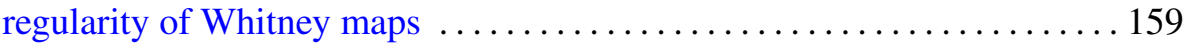

Elyahu Katz and Sidney Allen Morris, Free products of topological groups

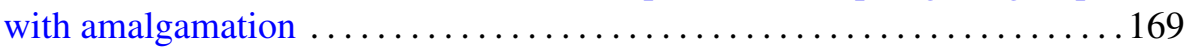

Kyewon Koh Park, Nice dense subsets for ergodic flows and Bernoulli

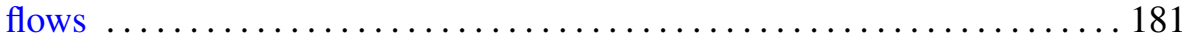

Francis Pastijn and Peter George Trotter, Lattices of completely regular

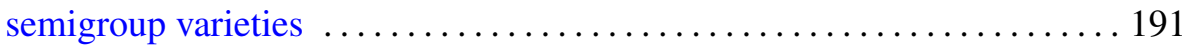

Rae Michael Andrew Shortt, Reticulated sets and the isomorphism of analytic powers

David A. Stegenga and Kenneth R. Stephenson, Generic covering properties for spaces of analytic functions

M. V. Subba Rao and R. Sitaramachandra Rao, On some infinite series of

L. J. Mordell and their analogues 